TRANSACTIONS OF THE

AMERICAN MATHEMATICAL SOCIETY

Volume 350, Number 11, November 1998, Pages 4521-4552

S 0002-9947(98)02006-6

\title{
SOLUTIONS OF NONLINEAR DIFFERENTIAL EQUATIONS ON A RIEMANNIAN MANIFOLD AND THEIR TRACE ON THE MARTIN BOUNDARY
}

\author{
E. B. DYNKIN AND S. E. KUZNETSOV
}

Abstract. Let $L$ be a second order elliptic differential operator on a Riemannian manifold $E$ with no zero order terms. We say that a function $h$ is $L$-harmonic if $L h=0$. Every positive $L$-harmonic function has a unique representation

$$
h(x)=\int_{E^{\prime}} k(x, y) \nu(d y),
$$

where $k$ is the Martin kernel, $E^{\prime}$ is the Martin boundary and $\nu$ is a finite measure on $E^{\prime}$ concentrated on the minimal part $E^{*}$ of $E^{\prime}$. We call $\nu$ the trace of $h$ on $E^{\prime}$.

Our objective is to investigate positive solutions of a nonlinear equation

$$
L u=u^{\alpha} \quad \text { on } E
$$

for $1<\alpha \leq 2$ [the restriction $\alpha \leq 2$ is imposed because our main tool is the $(L, \alpha)$-superdiffusion, which is not defined for $\alpha>2]$. We associate with every solution $u$ of $\left(^{*}\right)$ a pair $(\Gamma, \nu)$, where $\Gamma$ is a closed subset of $E^{\prime}$ and $\nu$ is a Radon measure on $O=E^{\prime} \backslash \Gamma$. We call $(\Gamma, \nu)$ the trace of $u$ on $E^{\prime} . \Gamma$ is empty if and only if $u$ is dominated by an $L$-harmonic function. We call such solutions moderate. A moderate solution is determined uniquely by its trace. In general, many solutions can have the same trace.

In an earlier paper, we investigated the case when $L$ is a second order elliptic differential operator in $\mathbb{R}^{d}$ and $E$ is a bounded smooth domain in $\mathbb{R}^{d}$. We obtained necessary and sufficient conditions for a pair $(\Gamma, \nu)$ to be a trace, and we gave a probabilistic formula for the maximal solution with a given trace.

The general theory developed in the present paper is applicable, in particular, to elliptic operators $L$ with bounded coefficients in an arbitrary bounded domain of $\mathbb{R}^{d}$, assuming only that the Martin boundary and the geometric boundary coincide.

\section{INTRODUCTION}

0.1 Definition of the trace. The key ingredients of this definition are: (1) a concept of a moderate solution and (2) operators $Q_{B}$ indexed by closed subsets of the Martin boundary $E^{\prime}$ and acting on positive solutions of the equation

$$
L u=u^{\alpha} .
$$

Received by the editors August 6, 1996.

1991 Mathematics Subject Classification. Primary 60J60, 35J60; Secondary 60J80, 60J45, 35J65.

Partially supported by National Science Foundation Grant DMS-9301315.

(C) 1998 American Mathematical Society 
A positive solution $u$ of $(0.1)$ is called moderate if it is dominated by an $L$ harmonic function. For such $u$, there exists the minimal $L$-harmonic majorant $h$. The trace of $u$ is defined as the finite measure $\nu$ which appears in the Martin integral representation of $h$.

We introduce the operators $Q_{B}$ probabilistically, but they can also be defined analytically. [The equivalence of both approaches follows from [6].] If $u$ is a solution of $(0.1)$ and $D$ is a regular open set in $E$, then there exists a minimal element $v_{D}$ among positive solutions $v$ of $(0.1)$ in $D$ such that, for every $c \in \partial D, v(x) \rightarrow u(c)$ as $x \rightarrow c$. Moreover, $v_{D}$ is monotone decreasing in $D$. Let $d(x, y)$ be the Martin distance between $x$ and $y$. For every closed subset $B$ of $E^{\prime}$, there exists a sequence of regular open sets $D_{n}$ in $E$ with the properties that for every $n, \inf _{x \in D_{n}} d(x, B)>0$ and $\sup d(x, B) \rightarrow 0$ as $n \rightarrow \infty$. The limit $\lim v_{D_{n}}$ exists, and it does not depend $x \notin D_{n}$ on the choice of $D_{n}$. We denote it by $Q_{B}(u)$.

The trace of a solution $u$ can be defined as a pair (a closed set $\Gamma$, a Radon measure $\nu$ on $O=E^{\prime} \backslash \Gamma$ ) with the following properties:

(i) For every closed $B \subset O, Q_{B}(u)$ is moderate and its trace coincides with $\nu$ on $B$.

(ii) If $Q_{B}(u)$ is moderate for $B \subset \Gamma$, then $Q_{B}(u)=0$.

0.2. Traces of moderate solutions. Which measures $\nu$ are the traces of moderate solutions? The answer to this question was given in [12] in the classical case when $L$ is an elliptic operator in $\mathbb{R}^{d}$ and $E$ is a bounded smooth domain in $\mathbb{R}^{d}$. We introduced a class of exceptional sets on the boundary and we proved that a finite measure $\nu$ is the trace of a moderate solution if and only if it does not charge exceptional sets. The class of exceptional sets $\Gamma$ is described by each of the following two equivalent definitions:

0.2.A. $\Gamma$ has the Martin capacity 0 , which means that if $\mu(\Gamma) \neq 0$, then

$$
\int_{E} \gamma(d x)\left[\int_{\Gamma} k(x, y) \mu(d y)\right]^{\alpha}=\infty .
$$

(Here $\gamma(d x)=g(c, x) d x, g(x, y)$ is the Green's function, $c$ is a reference point and $k(x, y)$ is the Martin kernel.)

0.2.B. $\Gamma$ is $\mathcal{R}$-polar, i.e. it is not hit by the range $\mathcal{R}$ of $(L, \alpha)$-superdiffusion (see definitions in Sections 1.2 and 1.3).

In the general case that we are studying now, the equivalence of definitions 0.2.A and 0.2.B is not proved. However we established in [8] that 0.2.B implies 0.2.A. It is natural to call sets which satisfy 0.2 .A weakly exceptional and sets which satisfy 0.2 .B strongly exceptional. We proved in [8] that the condition " $\nu$ does not charge weakly exceptional sets" is sufficient and the condition " $\nu$ does not charge strongly exceptional sets" is necessary for $\nu$ to be the trace of a moderate solution $u$.

0.3. Traces of arbitrary solutions. In Sections 6 and 7 we investigate the traces $(\Gamma, \nu)$ of arbitrary solutions of (0.1) under the assumption

$(\mathbb{N}) Q_{B}\left[Q_{\Gamma}(u)\right]=0$ for all solutions $u$ and all pairs of disjoint closed sets $B, \Gamma$.

In the classical case, $(\mathbb{N})$ holds by Theorem 3.1 in [12]. In Section 8 we establish more general conditions sufficient for $(\mathbb{N})$. [At present we have no examples when $(\mathbb{N})$ is false.]

The following two theorem are proved under assumption $(\mathbb{N})$. 
Theorem A. The trace $(\Gamma, \nu)$ of every solution u has the following properties:

0.3.A. $\nu$ does not charge strongly exceptional sets.

0.3.B. Every nonempty relatively open strongly exceptional subset $B$ of $\Gamma$ contains explosion points of $\nu$. of $c$.]

[We say that $c$ is an explosion point of $\nu$ if $\nu(U)=\infty$ for every neighborhood $U$

Theorem B. Suppose that $(\Gamma, \nu)$ satisfies 0.3.B and the following condition:

0.3. $A^{*} . \nu$ does not charge weakly exceptional sets.

Then $(\Gamma, \nu)$ is the trace of a solution.

0.4. On assumption $(\mathbb{N})$. In Section 8 we introduce the concept of a normal point at the Martin boundary $E^{\prime}$, and we prove that assumption $(\mathbb{N})$ is satisfied if all points are normal. The definition of a normal point is probabilistic, but we also give an analytic criterion of normality (the existence of an appropriate barriers).

The general theory is then applied to the case when $E$ is a bounded domain in $\mathbb{R}^{d}$ and $L$ is an elliptic operator in $E$. We show that $(\mathbb{N})$ holds if:

(a) the coefficients of $L$ are bounded;

(b) the Martin boundary $E^{\prime}$ coincides with the boundary $\partial E$ of $E$ in Euclidean geometry.

In fact, (a) can be replaced by a weaker condition 8.5.B and, instead of (b), it is sufficient to assume that one of the following two conditions is satisfied:

(a') The transition from $\partial E$ to $E^{\prime}$ does not lead to "pasting together" any pair of points.

(a") No "pasting" is needed for transition from $E^{\prime}$ to $\partial E$, and only a finite number of points of $E^{\prime}$ correspond to each point of $\partial E$.

[More precisely, put $c \hookrightarrow b$ if $c \in E^{\prime}, b \in \partial E$ and if there exists a sequence $x_{n} \in E$ such that $x_{n} \rightarrow c$ in the Martin topology and $x_{n} \rightarrow b$ in the Euclidean topology. Condition (a') means that $c \hookrightarrow b, c^{\prime} \hookrightarrow b$ implies $c=c^{\prime}$. Condition (a") means that $c \hookrightarrow b, c \hookrightarrow b^{\prime}$ implies $b=b^{\prime}$ and, for every $b$, there exist only a finite number of $c$ such that $c \hookrightarrow b$. ]

One of conditions (a'), (a") holds in all examples described in [1] to demonstrate distinctions between two boundaries.

0.5. Probabilistic solution. The solution described in Theorem B is defined by the formula

$$
u(x)=-\log P_{x}\left\{\mathcal{R} \cap \Gamma=\emptyset, e^{-A_{\nu}}\right\}
$$

where $\mathcal{R}$ is the range of $(L, \alpha)$-superdiffusion $\left(X, P_{x}\right)$ and $A_{\nu}$ is a continuous linear additive functional of $\left(X, P_{x}\right)$ with spectral measure $\nu$ (see definitions in Sections $1.2,1.3$ and 4.3). In Section 7 we prove that the solution (0.2) dominates all solutions with the same trace.

In general many solutions with the same trace can be constructed by applying formula (0.2) to various Borel sets $\Gamma \subset E^{\prime}$ with the same closure. [An example of nonuniqueness based on this idea was communicated to us by J.-F. Le Gall.]

0.6. Bibliographical notes. Open problems. A pair $(\Gamma, \nu)$ as a characteristic of a positive solution of the equation $\Delta u=u^{\alpha}$ first appeared in Le Gall's paper [13]. He proved that in the case of the unit disk $D$ in the plane and $\alpha=2$, there is a 1-1 correspondence between all positive solutions and all pairs $(\Gamma, \nu)$, where $\Gamma$ is a closed subset of $\partial D$ and $\nu$ is a Radon measure on $\partial D \backslash \Gamma$ (in this 
case, there exist no exceptional sets, and therefore conditions 0.3.A, 0.3.A' and 0.3.B hold automatically). For constructing a solution, Le Gall uses the Brownian snake - a path-valued Markov process that he discovered, closely related to $(\Delta, 2)$ superdiffusion. In [14] he extended the result to arbitrary planar domains of class $C^{2}$.

Marcus and Véron have announced in [15] and [16] and proved in [17] and [17a] results on the equation $\Delta u=u^{\alpha}$ in the unit ball of $\mathbb{R}^{d}$ for arbitrary $\alpha>1$ and $d$. [The name "trace" for a pair $(\Gamma, \nu)$ was first suggested in those publications.] By purely analytic means, they proved the existence and uniqueness of a solution with a given trace in the case $\alpha<(d+1) /(d-1)$ (where there exist no exceptional sets). In the case $\alpha \geq(d+1) /(d-1)$, they proved the existence of a solution under a condition on $\nu$ which is stronger than 0.3. $\mathrm{A}^{*}$.

The definition of the trace in [13], [14], [15] and [16] is different from ours. It is based on asymptotic behavior of a solution near the boundary.

The equation $L u=u^{\alpha}$ for an elliptic operator $L$ in $\mathbb{R}^{d}$ and a bounded domain of class $C^{2, \lambda}$ has been investigated in [12]. Necessary and sufficient conditions have been established for a pair $(\Gamma, \nu)$ to be a trace. (Note that in this case conditions 0.3.A and 0.3.A* are equivalent, and therefore the results in [12] follow from Theorems A and B.)

An important role was played in [12] by the characterization of $Q_{B}(u)$ as the maximal solution dominated by $u$ and equal to 0 on $\partial E \backslash \mathcal{B}$. (Condition $(\mathbb{N})$ is a direct implication of this fact.) Such a simple characterization is not available in the general setting. Among tools which we use to overcome this difficulty is a stochastic version of the comparison principle (see Theorem 2.1).

Several challenging problems remain open:

A. Do there exist any positive solutions of (0.1) which cannot be represented in the form (0.2) with a suitable coanalytic set $\Gamma$ and measure $\nu$ ?

$B$. Is it true in the general setting that the trace uniquely determines a solution if there are no exceptional sets on $E^{\prime}$ ?

C. Can the class of weakly exceptional sets be wider than the class of strongly exceptional sets?

\section{ACKNOWLEDGMENTS}

We are grateful to J.-F. Le Gall, M. Marcus and L. Véron for keeping us informed of their recent work.

\section{Diffusions AND SUPERDIFFUSIONS}

1.1. Diffusions. A general second order elliptic differential operator with no zero order terms on a Riemannian manifold $E$ has the form $L=\Delta+Y$, where $\Delta$ is the Laplace-Beltrami operator and $Y$ is a first order differential operator (i.e., a vector field). We denote by $m(d x)$ the measure on $E$ determined by the Riemannian metric. The $L$-diffusion is a Markov process $\xi=\left(\xi_{t}, \Pi_{x}\right)$ on $E$ with continuous paths and with the transition function $p_{t}(x, y) m(d y)$ where $p_{t}(x, y)$ satisfies the following conditions:

1.1.A. For all $t, x$,

$$
\int_{E} p_{t}(x, y) m(d y) \leq 1 .
$$


1.1.B. For all $s, t>0$ and all $x, z$,

$$
\int_{E} p_{s}(x, y) m(d y) p_{t}(y, z)=p_{s+t}(x, z) .
$$

1.1.C. For every bounded positive function $\varphi \in C^{1}(E)$,

$$
v_{t}(x)=\int_{E} p_{t}(x, y) \varphi(y) m(d y)
$$

is a solution of the boundary value problem

$$
\begin{aligned}
\frac{\partial v}{\partial t}=L v \quad \text { for } t>0, \\
v \rightarrow \varphi \quad \text { as } t \downarrow 0 .
\end{aligned}
$$

The existence of a function $p_{t}(x, y)$ subject to conditions 1.1.A-C has been proved in [19], and the existence of the corresponding $L$-diffusion follows from [2].

If $L=\Delta$, then the corresponding $L$-diffusion is called the Brownian motion on E.

Solutions of the equation $\Delta h=0$ are called harmonic functions. We use the name $L$-harmonic functions for solutions of the equation $L h=0$. Every positive $L$-harmonic function $h$ has a unique representation

$$
h(x)=\int_{E^{\prime}} k(x, y) \nu(d y),
$$

where $k$ is the Martin kernel and $\nu$ is a finite measure on the Martin boundary $E^{\prime}$ concentrated on the minimal part $E^{*}$ of $E^{\prime}$. We call $\nu$ the trace of $h$.

Recall that the Martin metric $d(x, y)$ in $E$ is defined in terms of $k$, that the exit space $\hat{E}$ is a compact metric space obtained by completion of $E$ with respect to $d$ and that $E^{\prime}=\hat{E} \backslash E$. We say $M$-limit, $M$-closure etc. when referring to the corresponding topology.

A path $\xi_{t}$ is defined on a random time interval $[0, \zeta)$. The $M$-limit

$$
\xi_{\zeta-}=\lim _{t \uparrow \zeta} \xi_{t}
$$

exists, a.s., ${ }^{1}$ and it belongs to $E^{*}$. Let $\sigma$ be the probability measure on $E^{\prime}$ which appears in the Martin representation (1.1) of the $L$-harmonic function $h=1$. Then for every positive Borel function $\varphi$ and for all $x$,

$$
\Pi_{x} \varphi\left(\xi_{\zeta_{-}}\right)=\int k(x, y) \varphi(y) \sigma(d y)
$$

[In other words, $\varphi(y) \sigma(d y)$ is the trace of $h(x)=\Pi_{x} \varphi\left(\xi_{\zeta_{-}}\right)$.] More generally, to every $L$-harmonic function $h$ there corresponds a measure $\Pi_{x}^{h}$ on the path space such that, for every $B \subset E^{\prime}$,

$$
\Pi_{x}^{h}\left\{\xi_{\zeta-} \in B\right\}=\int_{B} k(x, y) \nu(d y)
$$

( $\nu$ is the trace of $h$ ). For every stopping time $\tau$ and every positive Borel function $\varphi$,

$$
\Pi_{x}^{h}\left\{\varphi\left(\xi_{\tau}\right) 1_{\tau<\zeta}\right\}=\Pi_{x}(\varphi h)\left(\xi_{\tau}\right)
$$

\footnotetext{
${ }^{1}$ Writing "a.s." means $\Pi_{x}$-a.s. for all $x \in E$.
} 
Moreover,

$$
\Pi_{x}^{h}=\int \Pi_{x}^{y} \nu(d y)
$$

where $\Pi_{x}^{y}$ is the measure corresponding to the $L$-harmonic function $k(\cdot, y)$. [The measure $\frac{1}{h(x)} \Pi_{x}^{h}$ is called the $h$-transform of $\Pi_{x}$.]

We assume that the function

$$
g(x, y)=\int_{0}^{\infty} p_{t}(x, y) d t
$$

is finite ${ }^{2}$ for all $x \neq y$, and we call it Green's function. Green's operator acts on positive Borel functions by the formula

$$
G \varphi(x)=\int_{E} g(x, y) \varphi(y) m(d y) .
$$

Every domain $D$ in $E$ is a submanifold of $E$. The $L$-diffusion on $D$ can be obtained by killing the path at the first exit time $\tau=\inf \left\{t: \xi_{t} \notin D\right\}$ from $D$. We denote by $g_{D}$ and $G_{D}$ the corresponding Green's function and Green's operator.

We write $\partial D$ for the boundary of $D$ in the topology of $E$. We put $\bar{D}=D \cup \partial D$.

We say that $D_{n}$ is a standard sequence approximating $D$ if $D_{n}$ are open subsets of $D$ with compact closures $\bar{D}_{n}$ and if $\bar{D}_{n} \uparrow D$.

1.2. Superdiffusions. This is a mathematical model of a random cloud. The spatial motion of its infinitesimal parts is described by the $L$-diffusion $\xi=\left(\xi_{t}, \Pi_{x}\right)$, and the branching mechanism is determined by a parameter $\alpha \in(1,2]$. To every open set $D$ in $E$ and to every $\mu \in \mathcal{M}=\mathcal{M}(E)^{3}$ there corresponds a random measure $\left(X_{D}, P_{\mu}\right)$, called the exit measure from $D . X_{D}$ describes the mass distribution of the cloud instantaneously frozen on $E \backslash D$, and $P_{\mu}$ is a probability measure corresponding to the initial mass distribution $\mu$. All $P_{\mu}$ have the same domain $\mathcal{F}$. For every positive Borel function $f$,

$$
P_{\mu} \exp \left\langle-f, X_{D}\right\rangle=\exp \langle-u, \mu\rangle,
$$

where

$$
u+\mathcal{E}_{D}(u)=K_{D} f
$$

with

$$
\begin{gathered}
\mathcal{E}_{D}(u)(x)=G_{D}\left(u^{\alpha}\right)(x), \\
K_{D} f(x)=\Pi_{x} f\left(\xi_{\tau}\right)
\end{gathered}
$$

( $\tau$ is the first exit time of $\xi$ from $D$ ). ${ }^{4}$

We denote by $P_{x}$ the measure $P_{\delta_{x}}$ corresponding to Dirac's measure at the point $x$.

The joint probability distribution of $X_{D_{1}}, \ldots, X_{D_{n}}$ is determined by (1.9) and by the Markov property: for every positive $\mathcal{F}_{\supset D}$-measurable $Y$,

$$
P_{\mu}\left\{Y \mid \mathcal{F}_{\subset D}\right\}=P_{X_{D}} Y
$$

where $\mathcal{F}_{\subset D}$ is the $\sigma$-algebra generated by $X_{D_{1}}$ with $D_{1} \subset D$ and $\mathcal{F}_{\supset D}$ is the $\sigma$-algebra generated by $X_{D_{2}}$ with $D_{2} \supset D$.

\footnotetext{
${ }^{2}$ Otherwise $g(x, y)=\infty$ for all $x, y$ and $(*)$ has no positive solutions.

${ }^{3}$ We denote by $\mathcal{M}(S)$ the space of all finite measures on a measurable space $S$.

${ }^{4}$ The right side in (1.11) means $\int_{\tau<\zeta} f\left(\xi_{\tau}\right) d \Pi_{x}$.
} 
The existence of a family $\left(X_{D}, P_{\mu}\right)$ subject to conditions (1.9) and (1.12) is proved in [3].

It follows from (1.8)-(1.11) that

$$
P_{\mu}\left\langle f, X_{D}\right\rangle=\left\langle K_{D} f, \mu\right\rangle .
$$

1.3. Range and polar sets. Consider the class $\mathbb{C}$ of all closed random sets $C(\omega)$ with the property that every exit measure $X_{D}$ is concentrated, a.s., on $C$. There exists a minimal element of $\mathbb{C}$, and it is defined uniquely up to indistinguishability. We denote it by $\mathcal{R}$ and call it the range of $X$. A set $B \subset E^{\prime}$ is called $\mathcal{R}$-polar if

$$
P_{x}\{\mathcal{R} \cap B=\emptyset\}=1 \quad \text { for all } x \in D .
$$

1.4. Markov process $\left(X_{t}, P_{\mu}\right)$. Besides exit measures $X_{D}$ we also consider random measures $X_{t}$ which describe the mass distribution at a fixed time $t$. For every positive Borel function $f$,

$$
P_{\mu} \exp \left\langle-f, X_{t}\right\rangle=\exp \left\langle-v_{t}, \mu\right\rangle,
$$

where

$$
v_{t}(x)+\Pi_{x} \int_{0}^{t} v_{t-s}^{\alpha}\left(\xi_{s}\right) d s=\Pi_{x} f\left(\xi_{t}\right) .
$$

Random measures $\left(X_{t}, P_{\mu}\right)$ form a Markov process in the state space $\mathcal{M}$. This is a more traditional model of superdiffusion than the model described in Section 1.2.

$$
\text { 2. Equation } L u=u^{\alpha} \text {. Operators } V_{D}
$$

2.1. Class $\mathcal{U}(D)$. We denote by $\mathcal{U}(D)$ the class of all positive $u$ such that

$$
L u=u^{\alpha} \quad \text { in } D
$$

and we put $\mathcal{U}=\mathcal{U}(E)$. We have:

2.1.A. If $u+\mathcal{E}_{D}(u)$ is $L$-harmonic in $D$, then $u \in \mathcal{U}(D)$.

2.1.B. For every positive Borel $f$,

$$
u(x)=-\log P_{x} e^{-\left\langle f, X_{D}\right\rangle}
$$

belongs to $\mathcal{U}(D)$.

2.1.C. If $u_{n} \in \mathcal{U}(D)$ converge pointwise on $D$ to $u$, then $u \in \mathcal{U}(D)$.

2.1.D. (The mean value property) If $u \in \mathcal{U}$ and if $\bar{D}$ is compact, then

$$
u(x)=-\log P_{x} e^{-\left\langle u, X_{D}\right\rangle} \quad \text { in } D .
$$

2.1.A follows from Theorem 1.3 in [8]. By (1.8)-(1.9), 2.1.B follows from 2.1.A. Property 2.1.C is proved in [4, Theorem 1.2] and 2.1.D follows from Lemma 1.1 in [4] or Theorem 2.3 in [7].

2.2. Comparison principle. Let $\tau$ be the first exit time from an open set $D$. For every real-valued function $f$ on $D$, we set

$$
f^{D}=\limsup _{t \uparrow \tau} f\left(\xi_{t}\right) \quad \text { on }\{\tau>0\} .
$$

In particular,

$$
f^{E}=\limsup _{t \uparrow \zeta} f\left(\xi_{t}\right)
$$


If $B \subset \partial D$ and if $f$ is a continuous function on $D \cup B$, then

$$
f^{D}=f\left(\xi_{\tau}\right) \quad \text { on }\left\{\xi_{\tau} \in B, \tau>0\right\} .
$$

Lemma 2.1. Suppose that $\varphi$ is a bounded function on $\partial D$ and

$$
f(x)=\Pi_{x} \varphi\left(\xi_{\tau}\right) .
$$

Then

$$
f^{D}=\lim _{t \uparrow \tau} f\left(\xi_{t}\right)= \begin{cases}\varphi\left(\xi_{\tau}\right) & \text { a.s. } \text { on }\{0<\tau<\zeta\}, \\ 0 & \text { a.s. } \text { on }\{\tau=\zeta\} .\end{cases}
$$

If $\varphi \geq 0$ and

$$
f=G_{D} \varphi<\infty \text { on } D
$$

then

$$
f^{D}=0 \quad \text { a.s. on }\{\tau>0\} .
$$

Proof. If $f$ is given by $(2.5)$, then $f\left(\xi_{t \wedge \tau}\right)$ is a bounded continuous martingale, and therefore $\lim _{t \uparrow \tau} f\left(\xi_{t}\right)$ exists a.s. Clearly, it is equal to $f^{D}$. Let $\tau_{n}$ be the first exit times from a standard sequence $D_{n}$ approximating $D$. Then, a.s. on $\{0<\tau<\zeta\}$, $\tau_{n}<\tau$ and $\tau_{n} \rightarrow \tau$. Therefore $f^{D}=\lim f\left(\xi_{\tau_{n}}\right)$. On the other hand,

$$
f\left(\xi_{\tau_{n}}\right)=\Pi_{x}\left\{\varphi\left(\xi_{\tau}\right) \mid \mathcal{F}_{\tau_{n}}\right\} \rightarrow \varphi\left(\xi_{\tau}\right) \quad \text { a.s. on }\{0<\tau<\zeta\}
$$

because $\xi_{\tau}$ is measurable with respect to $\bigvee \mathcal{F}_{\xi_{\tau_{n}}}$. Besides,

$$
\Pi_{\xi_{\tau_{n}}}\{\tau<\zeta\}=\Pi_{x}\left\{\tau<\zeta \mid \mathcal{F}_{\tau_{n}}\right\} \rightarrow 0 \quad \text { a.s. on }\{\tau=\zeta\} .
$$

Formula (2.6) follows from (2.9) and (2.10). If $f$ is given by (2.7), then $f\left(\xi_{\tau \wedge t}\right)$ is a positive supermartingale and

$$
f^{D}=\lim _{t \uparrow \tau} f\left(\xi_{t}\right)=\lim _{t \uparrow \tau} \Pi_{\xi_{t}} \int_{0}^{\tau} \varphi\left(\xi_{s}\right) d s .
$$

Formula (2.8) follows from the relation

$$
\Pi_{x} \Pi_{\xi_{\tau_{n}}} \int_{0}^{\tau} \varphi\left(\xi_{s}\right) d s=\Pi_{x} \int_{\tau_{n}}^{\tau} \varphi\left(\xi_{s}\right) d s \rightarrow 0 \quad \text { a.s. on }\{0<\tau\} .
$$

Lemma 2.2. Suppose that $u \in C^{2}(D)$ is bounded above and

$$
L u \geq 0 \text { on } D .
$$

$$
u^{D} \leq 0 \quad \Pi_{x}-a . s .
$$

for all $x \in D$, then $u \leq 0$ in $D$.

Proof. Let $D_{n}$ be a standard sequence approximating $D$ and let $\tau_{n}$ be the first exit time from $D_{n}$. By Ito's formula,

$$
M_{t}=u\left(\xi_{\tau_{n} \wedge t}\right)-\int_{0}^{\tau_{n} \wedge t} L u\left(\xi_{s}\right) d s
$$

is a martingale relative to all measures $\Pi_{x}$. Therefore $\Pi_{x} M_{t}=\Pi_{x} M_{0}=u(x)$, and (2.11) implies $\Pi_{x} u\left(\xi_{\tau_{n} \wedge t}\right) \geq u(x)$. Since $\tau_{n} \uparrow \tau$ a.s., Fatou's lemma implies $\Pi_{x} u\left(\xi_{\tau \wedge t}\right) \geq u(x)$. By applying Fatou's lemma once more, we conclude that $\Pi_{x} u^{D} \geq$ $u(x)$ and $u \leq 0$ by (2.12). 
Theorem 2.1 (Comparison principle). Suppose $u, v \in C^{2}(D)$ satisfy the following conditions:

$$
L u-u^{\alpha} \leq L v-v^{\alpha} \quad \text { in } D
$$

$v-u$ is bounded above and

$$
(v-u)^{D} \leq 0 \quad \Pi_{x}-a . s .
$$

for all $x \in D$. Then $v \leq u$ in $D$.

Proof. Put $w=v-u$. Suppose $\tilde{D}=\{w>0\}$ is nonempty. Note that $L w=$ $L v-L u \geq v^{\alpha}-u^{\alpha}>0$ on $\tilde{D}$. Let $\tau$ and $\tilde{\tau}$ be the first exit times from $D$ and $\tilde{D}$. By $(2.4), w^{\tilde{D}}=w\left(\xi_{\tilde{\tau}}\right)=0$ a.s. on $\{\tilde{\tau}<\tau\}$. By $(2.14), w^{D}=0$ a.s. on $\{\tilde{\tau}=\tau\}$. It follows from Lemma 2.2 (applied to $\tilde{D}$ ) that $w \leq 0$ on $\tilde{D}$, which contradicts the definition of $\tilde{D}$.

2.3. Operators $V_{D}$. With every open set $D$ we associate an operator which acts on positive Borel functions on $E$ by the formula

$$
V_{D}(f)(x)=-\log P_{x} e^{-\left\langle f, X_{D}\right\rangle} .
$$

By 2.1.B, $V_{D}(f) \in \mathcal{U}(D)$. If $x \notin D$, then $P_{x}\left\{X_{D}=\delta_{x}\right\}=1$, and therefore $V_{D}(f)=f$ on $E \backslash D$. It follows from (1.8)-(1.9) that

$$
V_{D}(f) \leq K_{D} f
$$

The following properties of the operators $V_{D}$ are proved in [12, Section 2.2]:

2.3.A. $V_{D}\left(f_{1}\right) \leq V_{D}\left(f_{2}\right)$ for $f_{1} \leq f_{2}$.

2.3.B. If $D_{1} \subset D_{2}$, then $V_{D_{1}} V_{D_{2}}=V_{D_{2}}=V_{D_{2}} V_{D_{1}}$.

2.3.C. For every $f_{1}, f_{2}$,

$$
V_{D}\left(f_{1}+f_{2}\right) \leq V_{D}\left(f_{1}\right)+V_{D}\left(f_{2}\right) .
$$

2.3.D. For every $D_{1}, D_{2}$,

$$
V_{D_{1} \cap D_{2}}(f) \leq V_{D_{1}}(f)+V_{D_{2}}(f) \quad \text { in } D_{1} \cap D_{2} .
$$

\subsection{Action of $V_{D}$ on solutions.}

Theorem 2.2. If $u \in \mathcal{U}$, then

$$
V_{D}(u) \leq u
$$

If $D_{1} \subset D_{2}$, then

$$
V_{D_{2}}(u) \leq V_{D_{1}}(u) .
$$

Proof. Put $u_{B}=u 1_{B}$. First, we prove that, for every compact set $B \subset \partial D$, the function $w=V_{D}\left(u_{B}\right)$ has the properties

$$
w^{D}= \begin{cases}u_{B}\left(\xi_{\tau}\right) & \text { a.s. on }\{0<\tau<\zeta\}, \\ 0 & \text { a.s. on }\{\tau=\zeta\} .\end{cases}
$$

and

$$
w \leq u
$$

By (1.8)-(1.9), we have

$$
w+\mathcal{E}_{D}(w)=h \quad \text { in } D
$$


with $h(x)=\Pi_{x} u_{B}\left(\xi_{\tau}\right)$. By Lemma 2.1,

$$
\begin{aligned}
& h^{D}= \begin{cases}u_{B}\left(\xi_{\tau}\right) & \text { a.s. on }\{0<\tau<\zeta\}, \\
0 & \text { a.s. on }\{\tau=\zeta\} ;\end{cases} \\
& {\left[\mathcal{E}_{D}(w)\right]^{D}=0 \quad \text { a.s. on }\{\tau>0\}}
\end{aligned}
$$

and (2.21) implies (2.19). By (2.4) and (2.19), $u^{D}=u\left(\xi_{\tau}\right) \geq u^{B}\left(\xi_{\tau}\right)=w^{D}$ a.s. on $\{0<\tau<\zeta\}$ and $u^{D} \geq 0=w^{D}$ a.s. on $\{\tau=\zeta\}$. Hence, $(w-u)^{D} \leq 0$ a.s.

Since $w$ is bounded and $L u-u^{\alpha}=L w-w^{\alpha}=0$ in $D$, (2.20) follows from Theorem 2.1.

Consider compact sets $B_{n}$ such that $B_{n} \uparrow \partial D$, and let $w_{n}=V_{D}\left(u_{B_{n}}\right)$. Clearly, $w_{n} \uparrow V_{D}(u)$, and (2.20) implies (2.17).

If $D_{1} \subset D_{2}$, then, by 2.3.B, (2.17) and 2.3.A, $V_{D_{2}}(u)=V_{D_{1}} V_{D_{2}}(u) \leq V_{D_{1}}(u)$.

Remark. For every $c \geq 0$,

$$
V_{D}(c u) \leq(c \vee 1) u .
$$

If $c<1$, this follows from 2.3.A. If $c \geq 1$, then $L(c u)-(c u)^{\alpha} \leq 0$, and we get (2.22) by the arguments used in proof of $(2.17)$ with $w=V_{D}\left(c u_{B}\right)$.

\section{Probabilistic Representation of Solutions}

3.1. Stochastic boundary value of a solution $u$. Denote by $\mathcal{O}$ the class of all open sets $D$ in $E$ such that $\bar{D}$ is compact. Recall that according to Section 1.2, $\mathcal{F}_{\supset D}$ stands for the $\sigma$-algebra generated by $X_{\tilde{D}}$ with $\tilde{D} \supset D$. We use the name the germ $\sigma$-algebra of $X$ on the Martin boundary for the intersection $\mathcal{F}\left(E^{\prime}\right)$ of $\mathcal{F}_{\supset D}$ over all $D \in \mathcal{O}$. Note that

$$
\mathcal{F}\left(E^{\prime}\right)=\bigcap_{n} \mathcal{F}_{\supset D_{n}}
$$

for every standard sequence $D_{n}$ approximating $E$.

Theorem 3.1. For every $u \in \mathcal{U}$, there exists a $\mathcal{F}\left(E^{\prime}\right)$-measurable function $Z \geq 0$ such that

$$
\lim \left\langle u, X_{D_{n}}\right\rangle=Z \quad P_{\mu} \text {-a.s. }
$$

for every standard sequence $D_{n}$ approximating $E$ and every $\mu \in \mathcal{M}$. For all $\mu \in \mathcal{M}$,

$$
P_{\mu} e^{-Z}=e^{-\langle u, \mu\rangle} \text {. }
$$

In particular,

$$
u(x)=-\log P_{x} e^{-Z} \quad \text { for all } x \in E .
$$

Proof. Suppose that $D_{n}$ is a standard sequence approximating $E$ and put $Y_{n}=$ $e^{-\left\langle u, X_{D_{n}}\right\rangle}$. By (1.12), (1.8), (2.15) and (2.17),

$$
P_{\mu}\left\{Y_{n+1} \mid \mathcal{F}_{\subset D_{n}}\right\}=P_{X_{D_{n}}} Y_{n+1}=e^{-\left\langle V_{D_{n+1}}(u), X_{D_{n}}\right\rangle} \geq Y_{n}
$$

Hence $\left(Y_{n}, \mathcal{F}_{\subset D_{n}}, P_{\mu}\right)$ is a bounded submartingale, which implies the existence, $P_{\mu}$-a.s., of $\lim \left\langle u, X_{D_{n}}\right\rangle$.

Fix a standard sequence $D_{n}^{0}$ approximating $E$ and put $Z=\lim \sup \left\langle u, X_{D_{n}^{0}}\right\rangle$. If $D_{n}$ is an arbitrary standard sequence, then there exist $n_{1}<n_{2}<\cdots$ such that

$$
D_{n_{1}}^{0} \subset D_{n_{2}} \subset D_{n_{3}}^{0} \subset D_{n_{4}} \subset \cdots .
$$


There exists, $P_{\mu}$-a.s., a limit of $\left\langle u, X_{D}\right\rangle$ along the standard sequence (3.5). It coincides, $P_{\mu}$-a.s., with the limit along $D_{n}$ and the limit along $D_{n}^{0}$, which proves (3.2). Formula (3.3) follows from (3.2) and the dominated convergence theorem.

We call $Z$ defined in Theorem 3.1 the stochastic boundary value of $u$. (If two functions $Z_{1}$ and $Z_{2}$ satisfy this definition, then $Z_{1}=Z_{2} P_{\mu}$-a.s. for all $\mu \in \mathcal{M}$.) The function $u$ defined by formula (3.4) is called the log-potential of $Z$. Theorem 3.1 can be restated as follows:

Theorem 3.1 bis. For every $u \in \mathcal{U}$, there exists a stochastic boundary value $Z$. The log-potential of $Z$ is equal to $u$.

3.2. Total classes of measures. We say that a subset $\mathcal{M}^{*}$ of a set $\mathcal{M}$ is total if: 3.2.A. If $\mu \in \mathcal{M}^{*}$ and if $\tilde{\mu} \leq \mu$, then $\tilde{\mu} \in \mathcal{M}^{*}$.

3.2.B. For every $\mu \in \mathcal{M}^{*}$ and for an arbitrary $D \in \mathcal{O}, P_{\mu}\left\{X_{D} \in \mathcal{M}^{*}\right\}=1$.

3.2.C. $\mathcal{M}^{*}$ contains all Dirac's measures $\delta_{x}, x \in E$.

Clearly, the intersection of any countable family of total sets is a total set.

To an arbitrary $L$-harmonic function $h$ there corresponds a total set $\mathcal{M}^{*}(h)=$ $\{\mu \in \mathcal{M}:\langle h, \mu\rangle\langle\infty\rangle\}$. The class $\mathcal{M}_{\mathcal{E}}^{*}(h)=\langle h+\mathcal{E}(h), \mu\rangle<\infty$ satisfies 3.2.A, B. If $\mathcal{E}(h)(x)<\infty$ for some $x$, then $\mathcal{E}(h)(x)<\infty$ for all $x$ (this follows from Harnack's inequality). Hence, $\mathcal{M}_{\mathcal{E}}^{*}(h)$ satisfies 3.2.C and is a total class.

One more example of a total set: put $\mu \in \mathcal{M}_{0}^{*}$ if $\mu \in \mathcal{M}$ and if $\mu(E \backslash D)=0$ for some $D \in \mathcal{O}$. Clearly, $\mathcal{M}_{0}^{*}$ satisfies 3.2.A and C. By [5, I.9.A], it satisfies 3.2.B.

Two functions $Z_{1}$ and $Z_{2}$ are called $\mathcal{M}^{*}$-equivalent if $P_{\mu}\left\{Z_{1} \neq Z_{2}\right\}=0$ for all $\mu \in \mathcal{M}^{*}$.

3.3. Class $\mathfrak{Z}\left(\mathcal{M}^{*}\right)$. Let $\mathcal{M}^{*}$ be a total set. Denote by $\mathcal{F}\left(E^{\prime}, \mathcal{M}^{*}\right)$ the completion of $\mathcal{F}\left(E^{\prime}\right)$ with respect to all $P_{\mu}, \mu \in \mathcal{M}^{*}$. The class $\mathfrak{Z}\left(\mathcal{M}^{*}\right)$ consists of all positive $\mathcal{F}\left(E^{\prime}, \mathcal{M}^{*}\right)$-measurable functions $Z$ subject to the conditions:

$\mathrm{CB}\left(\mathcal{M}^{*}\right) .{ }^{5}$ For every $\mu \in \mathcal{M}^{*}$,

$$
-\log P_{\mu} e^{-Z}=\int\left[-\log P_{x} e^{-Z}\right] \mu(d x) .
$$

and

FIN. For all $x \in E, P_{x}\{Z<\infty\}>0$.

We use the shorter notation $\mathfrak{Z}$ for $\mathfrak{Z}(\mathcal{M})$.

Theorem 3.2. A stochastic boundary value $Z$ of any $u \in \mathcal{U}$ belongs to $\mathfrak{Z}$. Let $Z \in \mathfrak{Z}\left(\mathcal{M}^{*}\right)$. Then the log-potential $u$ of $Z$ belongs to $\mathcal{U}$, and $Z$ is $\mathcal{M}^{*}$-equivalent to a stochastic boundary value of $u$.

Proof. $1^{\circ}$. The stochastic boundary value is $\mathcal{F}\left(E^{\prime}\right)$-measurable. Property $\mathrm{CB}(\mathcal{M})$ follows from (3.3) and (3.4). Since $u(x)<\infty$ for all $x \in E$, (3.4) implies FIN.

$2^{\circ}$. Let $u$ be the log-potential of $Z \in \mathfrak{Z}\left(\mathcal{M}^{*}\right)$. By FIN, $u<\infty$. Suppose $\mu \in \mathcal{M}^{*}$. By the Markov property (1.12),

$$
P_{\mu}\left\{e^{-Z} \mid \mathcal{F}_{\subset D}\right\}=P_{X_{D}} e^{-Z} \quad P_{\mu} \text {-a.s. }
$$

for every $D \in \mathcal{O}$. Then, by $\mathrm{CB}\left(\mathcal{M}^{*}\right)$ and 3.2.B,

$$
P_{X_{D}} e^{-Z}=e^{-\left\langle u, X_{D}\right\rangle} \quad P_{\mu} \text {-a.s. }
$$

\footnotetext{
${ }^{5} \mathrm{CB}$ stands for "continuous branching".
} 
Let $D_{n}$ be a standard sequence approximating $E$. All exit measures $X_{D}, D \in \mathcal{O}$, are measurable relative to the $\sigma$-algebra $\bigvee_{n} \mathcal{F}_{\subset D_{n}}$. Therefore $Z$ is also measurable with respect to this $\sigma$-algebra and, by (3.1), (3.7) and (3.8),

$$
e^{-Z}=\lim P_{\mu}\left\{e^{-Z} \mid \mathcal{F}_{\subset D_{n}}\right\}=\lim e^{-\left\langle u, X_{D_{n}}\right\rangle} \quad P_{\mu} \text {-a.s. },
$$

which implies

$$
\lim \left\langle u, X_{D_{n}}\right\rangle=Z \quad P_{\mu} \text {-a.s. }
$$

It follows from (3.4) and (3.9) that

$$
u(x)=\lim u_{n}(x),
$$

where

$$
u_{n}=V_{D_{n}}(u) \in \mathcal{U}\left(D_{n}\right) .
$$

By 2.1.C, $u \in \mathcal{U}$. By (3.9), $Z$ is $\mathcal{M}^{*}$-equivalent to a stochastic boundary value of $u$ determined by (3.2).

It follows from Theorem 3.2 that, for every $Z \in \mathfrak{Z}\left(\mathcal{M}^{*}\right)$, there exists an $\mathcal{M}^{*}$ equivalent function $\tilde{Z} \in \mathfrak{Z}$.

We denote by $\overline{\mathfrak{Z}}\left(\mathcal{M}^{*}\right)$ the set obtained from $\mathfrak{Z}\left(M^{*}\right)$ by identifying $\mathcal{M}^{*}$-equivalent functions. Theorem 3.2 implies:

Theorem 3.2 bis. Formula (3.4) defines a 1-1 mapping from $\overline{\mathfrak{Z}}\left(\mathcal{M}^{*}\right)$ onto $\mathcal{U}$. The inverse mapping is given by (3.9).

\subsection{Properties of log-potential.}

Lemma 3.1. Suppose $Z_{1}, Z_{2} \in \mathfrak{Z}\left(\mathcal{M}^{*}\right)$ and let $u_{1}, u_{2}, u$ be the log-potentials of $Z_{1}, Z_{2}$ and $Z=Z_{1}+Z_{2}$. Then

$$
u \leq u_{1}+u_{2} .
$$

Proof. Proposition 2.3.C and Theorem 2.2 imply that if $u_{1}, u_{2} \in \mathcal{U}$, then, for every $D$,

$$
V_{D}\left(u_{1}+u_{2}\right) \leq u_{1}+u_{2} .
$$

Let $x \in E$. By (3.2),

$$
Z=\lim \left\langle u_{1}+u_{2}, X_{D_{n}}\right\rangle \quad P_{x^{-}} \text {a.s. }
$$

By (3.12), (2.15) and by the dominated convergence theorem,

$$
u(x)=\lim V_{D_{n}}\left(u_{1}+u_{2}\right)(x) .
$$

Formula (3.10) follows from (3.11) and (3.13).

Theorem 3.3. If $\mathcal{M}^{*} \subset \mathcal{M}_{0}^{*}$, then $\mathfrak{Z}\left(\mathcal{M}^{*}\right)$ is a convex cone. The log-potential is a subadditive function on this cone.

Proof. Suppose $Z_{1}, Z_{2} \in \mathfrak{Z}\left(\mathcal{M}^{*}\right)$ and let $Z=c_{1} Z_{1}+c_{2} Z_{2}$, where $c_{1}, c_{2}$ are positive constants. Denote by $u_{1}, u_{2}, u$ the log-potentials of $Z_{1}, Z_{2}, Z$. Let $\mu \in \mathcal{M}^{*}$. It follows from (3.2) that

$$
Z=\lim \left\langle c_{1} u_{1}+c_{2} u_{2}, X_{D_{n}}\right\rangle \quad P_{\mu} \text {-a.s. }
$$

which implies

$$
-\log P_{\mu} e^{-Z}=\lim \left\langle V_{D_{n}}\left(c_{1} u_{1}+c_{2} u_{2}\right), \mu\right\rangle .
$$


Formula (3.15) applied to $\delta_{x}$ yields

$$
-\log P_{x} e^{-Z}=\lim V_{D_{n}}\left(c_{1} u_{1}+c_{2} u_{2}\right)(x) .
$$

It follows from 2.3.C and (2.22) that

$$
V_{D_{n}}\left(c_{1} u_{1}+c_{2} u_{2}\right) \leq\left(c_{1} \vee 1\right) u_{1}+\left(c_{2} \vee 1\right) u_{2} .
$$

Since $\mu \in \mathcal{M}^{*} \subset \mathcal{M}_{0}^{*}$, the right side in (3.17) is finite and, by the dominated convergence theorem,

$$
\int \lim V_{D_{n}}\left(c_{1} u_{1}+c_{2} u_{2}\right)(x) \mu(d x) \leq \lim \left\langle V_{D_{n}}\left(c_{1} u_{1}+c_{2} u_{2}\right), \mu\right\rangle .
$$

By (3.15), (3.16), (3.18) and (3.17), condition $\mathrm{CB}\left(\mathcal{M}^{*}\right)$ holds for $Z$. By (3.16) and (3.17), $Z$ satisfies FIN. Hence $Z \in \mathfrak{Z}\left(\mathcal{M}^{*}\right)$. By (3.10), $u$ is a subadditive function.

Theorem 3.4. Let $\mathcal{M}_{n}^{*}$ be total classes and let $\mathcal{M}^{*}$ be the intersection of the $\mathcal{M}_{n}^{*}$. Suppose $Z_{n} \in \mathfrak{Z}\left(\mathcal{M}_{n}^{*}\right)$ and $Z_{n} \uparrow Z P_{\mu}$-a.s. for all $\mu \in \mathcal{M}^{*}$. Then $Z \in \mathfrak{Z}\left(\mathcal{M}^{*}\right)$, and the log-potential $u_{n}$ of $Z_{n}$ converges to the log-potential $u$ of $Z$.

Proof. Clearly, $Z \in \mathcal{F}\left(E^{\prime}, \mathcal{M}^{*}\right)$. If $\mu \in \mathcal{M}^{*}$, then (3.6) holds for all $Z_{n}$ and, by the monotone convergence theorem and the dominated convergence theorem, it holds for $Z$. By the dominated convergence theorem, $u_{n} \uparrow u$. By Theorem 3.2, $u_{n} \in \mathcal{U}$ and, by 2.1.C, $u \in \mathcal{U}$. Since $u<\infty, Z$ satisfies FIN.

\section{Solutions Determined By CONTINUOUS Linear AdDitive FUnCTIONALS.}

$$
(\Gamma, \nu) \text {-SOLUTiONS }
$$

4.1. Continuous linear additive functionals. Let $\left(X_{t}, P_{\mu}\right)$ be a superdiffusion in $E$ and let $\mathcal{M}^{*}$ be a total subset of $\mathcal{M}$. Denote by $\mathcal{F}$ the $\sigma$-algebra generated by $X_{s}, s<\infty$, and by $\mathcal{F}_{t}$ the $\sigma$-algebra generated by $X_{s}, s \leq t$. A function $A_{t}(\omega)$ from $[0, \infty] \times \Omega$ to $[0, \infty]$ is called a continuous additive functional of $X$ with determining set $\mathcal{M}^{*}$ if:

4.1.A. $A_{0}=0$.

4.1.B. For every $t$ and every $\mu, A_{t}$ is measurable with respect to the $P_{\mu}$-completion of $\mathcal{F}$; it is also measurable with respect to $P_{\mu}$-completion of $\mathcal{F}_{t}$ if $\mu \in \mathcal{M}^{*}$.

4.1.C. $A_{s+t}=A_{s}+\theta_{s} A_{t} P_{\mu}$-a.s. for all $\mu \in \mathcal{M}^{*}$ and all pairs $s$, $t$. (Here $\theta_{s}$ are the shift operators for $X$.)

4.1.D. $A_{t}$ is continuous in $t$ for $P_{\mu}$-almost all $\omega$ for every $\mu \in \mathcal{M}^{*}$.

If the $A^{n}$ are continuous additive functionals with determining sets $\mathcal{M}_{n}^{*}$, then $A_{t}=A_{t}^{1}+\cdots+A_{t}^{n}+\ldots$ is a continuous additive functional with determining set $\cap \mathcal{M}_{n}^{*}$

Let $A$ and $\tilde{A}$ be continuous additive functionals with determining sets $\mathcal{M}^{*}$ and $\tilde{\mathcal{M}}^{*}$. We say that functionals $A$ and $\tilde{A}$ are equivalent and we write $A \sim \tilde{A}$ if $P_{\mu}\left\{A_{t}=\tilde{A}_{t}\right\}=1$ for all $t$ and all $\mu \in \mathcal{M}^{*} \cap \tilde{\mathcal{M}}^{*}$. We say that $A$ and $\tilde{A}$ are indistinguishable if, in addition, $\mathcal{M}^{*}=\tilde{\mathcal{M}}^{*}$.

We call

$$
h(x)=P_{x} A_{\infty}
$$

the potential and

$$
u(x)=-\log P_{x} e^{-A_{\infty}}
$$


the log-potential of $A$. [In the terminology of Section 3.1, $u$ is the log-potential of $A_{\infty}$.]

We say that a continuous additive functional $A$ is linear if

$$
P_{\mu} A_{\infty}=\langle h, \mu\rangle
$$

and

$$
P_{\mu} e^{-A_{\infty}}=e^{-\langle u, \mu\rangle}
$$

for all $\mu$ in determining set $\mathcal{M}^{*}$. [Formula (4.4) means that $A_{\infty}$ satisfies $\operatorname{CB}\left(\mathcal{M}^{*}\right)$.]

Suppose that the potential $h$ of a continuous linear additive (CLA) functional $A$ is an $L$-harmonic function $h$. It follows from Theorem 1.2 in [10] that the logpotential $u$ of $A$ satisfies equation

$$
u+\mathcal{E}(u)=h \quad \text { in } E,
$$

where $\mathcal{E}(u)=\mathcal{E}_{E}(u)=G\left(u^{\alpha}\right)$ (cf. (1.10)). Moreover, by Theorem 2.1 in [9], $u$ is the unique solution of (4.5). By 2.1.A, $u \in \mathcal{U}$.

4.2. Classes $H^{*}$ and $H^{* *}$. Denote by $H^{*}$ the class of all positive $L$-harmonic functions $h$ such that equation (4.5) has a positive solution $u$. Put $h \in H^{* *}$ if $h \in H^{*}$ and if $\mathcal{E}(h)(x)<\infty$ for some $x$ (which implies that $\mathcal{E}(h)(x)<\infty$ for all $x$ ). It follows from [8, Theorem 3.1 and proof of Theorem 2.2] (cf. [10, 4.1.B]) that every $h \in H^{*}$ can be represented in the form

$$
h=h_{1}+\cdots+h_{n}+\ldots \quad \text { with } h_{n} \in H^{* *} .
$$

Theorems 1.7, 1.1, 1.3 in [11] imply

Theorem 4.1. If $h \in H^{* *}$, then there exists a unique (up to indistinguishability) $C L A$ functional $A$ of $X$ with potential $h$ and determining set $\mathcal{M}_{\mathcal{E}}(h)$. The logpotential $u$ of $A$ is a unique solution of equation (4.5).

Remark. If $A^{n}$ are CLA functionals corresponding to $h_{n} \in H^{* *}$ by Theorem 4.1 and if $h=h_{1}+\cdots+h_{n}+\cdots \in H^{* *}$, then $A^{1}+\cdots+A^{n}+\ldots$ is equivalent to the CLA functional corresponding to $h$.

4.3. Continuous linear additive functional with spectral measure $\nu$. Denote by $\mathcal{N}^{*}$ the set of all measures $\nu$ on $E^{\prime}$ such that $K \nu \in H^{*}$. Analogously, $\nu \in \mathcal{N}^{* *}$ if $K \nu \in H^{* *}$. Put $\nu \in \mathcal{N}$ if there exist $\nu_{n} \in \mathcal{N}^{* *}$ such that

$$
\nu=\nu_{1}+\cdots+\nu_{n}+\ldots
$$

By $(4.6), \mathcal{N} \supset \mathcal{N}^{*}$.

Remark 1. Clearly, every $\nu \in \mathcal{N}$ is $\Sigma$-finite.

Remark 2. If $\nu^{n} \in \mathcal{N}^{* *}$ and $\nu^{n} \uparrow \nu \in \mathcal{N}^{* *}$, then, by the Remark to Theorem 4.1, $\lim A^{\nu_{n}} \sim A^{\nu}$.

Theorem 4.2. It is possible to define for every $\nu \in \mathcal{N}$ a CLA functional $A^{\nu}$ of $X$ with determining set $\mathcal{M}^{*}(\nu)$ in such a way that:

4.3.A. If $\nu=\nu_{1}+\cdots+\nu_{n}+\ldots$, then $A^{\nu}$ is equivalent to $A^{\nu_{1}}+\cdots+A^{\nu_{n}}+\ldots$ 4.3.B. If $\nu \in \mathcal{N}^{* *}$ and if $h=K \nu$, then $A^{\nu}$ is indistinguishable from the CLA functional corresponding to $h=K \nu$ by Theorem 4.1. 
Proof. $1^{\circ}$. We use Theorem 4.1 to define $A^{\nu}$ for $\nu \in \mathcal{N}^{* *}$, and we define $A^{\nu}$ as the sum of the $A^{\nu_{n}}$ if $\nu$ is defined by (4.7). To justify this definition, we prove that, if $\nu=\sum \nu_{i}=\sum \tilde{\nu}_{j}$, then $\sum A^{\nu_{i}} \sim \sum A^{\tilde{\nu}_{j}}$. Indeed, since the measure $\nu$ is $\Sigma$-finite, there exists a finite measure $\mu$ such that $\nu(d x)=\rho(x) \mu(d x)$ for a positive function $\rho$. Put

$$
\nu^{m}=\nu_{1}+\cdots+\nu_{m}, \quad \tilde{\nu}^{n}=\tilde{\nu}_{1}+\cdots+\tilde{\nu}_{n} .
$$

By the Radon-Nikodým theorem, $\nu^{m}(d x)=\rho^{m}(x) \mu(d x), \tilde{\nu}^{n}(d x)=\tilde{\rho}^{n}(x) \mu(d x)$ with $\lim \rho^{m}=\lim \tilde{\rho}^{n}=\rho \mu$-a.e. The measures $\nu^{m n}(d x)=\left[\rho^{m}(x) \wedge \tilde{\rho}^{n}(x)\right] \mu(d x)$ belong to $\mathcal{N}^{* *}$. The corresponding functionals $A^{m n}=A^{\nu^{m n}}$ are monotone increasing in $m, n$, and therefore

$$
\lim _{m} \lim _{n} A^{m n}=\sup _{m, n} A^{m n}=\lim _{n} \lim _{m} A^{m n} .
$$

Since $\nu^{m n} \uparrow \nu^{m}$ as $n \rightarrow \infty$, we have $\lim _{n} A^{m n} \sim A^{\nu^{m}}$ by Remark 2. Clearly, $A^{\nu^{m}} \sim A^{\nu_{1}}+\cdots+A^{\nu_{m}}$, and therefore the left side in (4.8) is equal to $\sum A^{\nu_{i}}$. Analogously, the right side is equal to $\sum \tilde{A}^{\nu_{j}}$.

$2^{\circ}$. Properties 4.3.A and B hold for $\nu \in \mathcal{N}^{* *}$ by Theorem 4.1, and they can be obtained for $\nu \in \mathcal{N}$ by a passage to the limit.

We call $A^{\nu}$ the $C L A$ functional with spectral measure $\nu$. If $\nu$ has the form (4.7), then $\bigcap \mathcal{M}_{\mathcal{E}}^{*}\left(\nu_{n}\right)$ is a determining set of $A^{\nu}$.

A necessary condition for a measure $\nu$ to belong to $\mathcal{N}$ is given by

Theorem 4.3. No measures $\nu \in \mathcal{N}$ charge $\mathcal{R}$-polar sets.

Proof. For finite measures $\nu$, this follows from [8, Theorem 3.1]. An extension to arbitrary $\nu \in \mathcal{N}$ is obvious because all $\nu \in \mathcal{N}$ are $\Sigma$-finite.

Theorem 4.4. If $A^{\nu}$ is a CLA functional with the spectral measure $\nu$ and if $\mathcal{M}^{*}$ is the determining set of $A^{\nu}$, then $A_{\infty}^{\nu}$ is an element of class $\mathfrak{Z}\left(\mathcal{M}^{*}\right)$, and the log-potential of $A^{\nu}$ belongs to $\mathcal{U}$.

Proof. By Theorem 3.4, it is sufficient to consider $\nu \in \mathcal{N}^{* *}$. Let $h$ and $u$ be the potential and the log-potential of $A^{\nu}$, and let $\mu \in \mathcal{M}^{*}$. By Lemma 5.2 in [10], $A_{\infty}^{\nu}$ is measurable with respect to the $P_{\mu}$-completion of $\mathcal{F}_{\supset D}$ for every $D \in \mathcal{O}$ and it is measurable with respect to the $P_{\mu}$-completion of $\bigcap \mathcal{F}_{\supset D_{n}}$ for every standard sequence $D_{n}$ approximating $E$. By Theorem 1.2 in [10], $u \in \mathcal{U}$. By (1.12) and (4.4), $P_{\mu}$-a.s.,

$$
P_{\mu}\left\{e^{-A_{\infty}^{\nu}} \mid \mathcal{F}_{\subset D_{n}}\right\}=P_{X_{D_{n}}} e^{-A_{\infty}^{\nu}}=e^{-\left\langle u, X_{D_{n}}\right\rangle}
$$

and therefore

$$
e^{-A_{\infty}^{\nu}}=e^{-\lim \left\langle u, X_{D_{n}}\right\rangle}
$$

By comparing this formula with (3.2), we note that $A_{\infty}^{\nu}$ is $\mathcal{M}^{*}$-equivalent to a stochastic boundary value of $u$. It belongs to $\mathfrak{Z}\left(\mathcal{M}^{*}\right)$ by Theorem 3.2.

4.5. $(\Gamma, \nu)$-solutions. For every closed set $\Gamma \subset E^{\prime}$ and every $\nu \in \mathcal{N}$ we set

$$
u(x)=-\log P_{x}\left\{\mathcal{R} \cap \Gamma=\emptyset, e^{-A_{\infty}^{\nu}}\right\} .
$$

We prove that $u \in \mathcal{U}$, and we call $u$ the $(\Gamma, \nu)$-solution.

We need 
Lemma 4.1. Let

$$
U_{n}=\{x: d(x, \Gamma)>1 / n\} .
$$

Then, for every $\mu \in \mathcal{M}$,

$$
\left\{X_{U_{n}}=0\right\} \uparrow\{\mathcal{R} \cap \Gamma=\emptyset\} \quad P_{\mu} \text {-a.s. }
$$

Proof. It follows from (1.12) that, $P_{\mu}$-a.s.,

$$
\left\{X_{U_{n}}=0\right\} \subset\left\{X_{U_{n+1}}=0\right\} .
$$

By [3, Lemma 2.1],

$$
\left\{\mathcal{R} \subset \bar{U}_{n-1}\right\} \subset\left\{X_{U_{n}}=0\right\} \subset\left\{\mathcal{R} \subset \bar{U}_{n}\right\} \quad P_{\mu} \text {-a.s. }
$$

Clearly, this implies (4.11).

Theorem 4.5. If $\Gamma$ is a closed subset of $E^{\prime}$, then

$$
Z(\Gamma)=\left\{\begin{array}{lll}
0 & \text { if } & \mathcal{R} \cap \Gamma=\emptyset \\
\infty & \text { if } & \mathcal{R} \cap \Gamma \neq \emptyset
\end{array}\right.
$$

belongs to class $\mathfrak{Z}\left(\mathcal{M}_{0}^{*}\right)$.

If $A^{\nu}$ is a CLA functional with spectral measure $\nu$ and determining set $\mathcal{M}^{*}$, then

$$
Z=Z(\Gamma)+A_{\infty}^{\nu}
$$

belongs to $\mathfrak{Z}\left(\mathcal{M}_{1}^{*}\right)$, where $\mathcal{M}_{1}^{*}=\mathcal{M}_{0}^{*} \cap \mathcal{M}^{*}$.

Proof. For every $D \in \mathcal{O}$, there exists $m$ such that $U_{n} \supset D$ for all $m \geq n$. By (4.11), $Z(\Gamma) \in \mathcal{F}\left(E^{\prime}, \mathcal{M}\right)$. Condition FIN holds for $Z(\Gamma)$, because $P_{x}\left\{\mathcal{R} \cap E^{\prime}=\emptyset\right\}>0$ for every $x$ by [4, Theorem 2.1]. Condition $\operatorname{CB}(\mathcal{M})$ holds for $Z_{n}=0$ if $X_{U_{n}}=0$ and $Z_{n}=\infty$ otherwise by [5, II.4.6]. Condition $\operatorname{CB}\left(\mathcal{M}_{0}^{*}\right)$ for $Z(\Gamma)$ follows from (4.11) and the dominated convergence theorem.

The second statement of Theorem 4.5 is an implication of the first statement and Theorem 3.3.

\section{Trace of SOlutions}

5.1. Moderate solutions. We say that $u \in \mathcal{U}$ is a moderate solution if it is dominated by an $L$-harmonic function. It follows from Theorem 1.3 in [8] and 2.1. A that:

5.1.A. If $u$ is a moderate solution, then $h=u+\mathcal{E}(u)$ is the minimal $L$-harmonic majorant of $u$ and $u$ is the maximal solution dominated by $h$.

The trace of a moderate solution $u$ is defined as the trace of the $L$-harmonic function $h=u+\mathcal{E}(u)$. By [8, Theorem 3.1],

5.1.B. If $\nu$ is the trace of a moderate solution $u$, then $\nu(B)=0$ for all $\mathcal{R}$-polar sets $B$.

5.1.C. The log-potential $u$ of $Z \in \mathfrak{Z}\left(\mathcal{M}^{*}\right)$ is a moderate solution if and only if

$$
h(x)=P_{x} Z<\infty \quad \text { for all } x \in E .
$$

If $u$ is moderate, then its minimal $L$-harmonic majorant is given by (5.1). 
Proof. For every $D \in \mathcal{O}$, by (1.12) and (1.13),

$$
h(x)=P_{x} P_{X_{D}} Z=P_{x}\left\langle h, X_{D}\right\rangle=\Pi_{x} h\left(\xi_{\tau}\right),
$$

where $\tau$ is the first exit time from $D$. Therefore, if $h<\infty$, then $h$ is $L$-harmonic. By Jensen's inequality, $P_{x} e^{-Z} \geq e^{-h(x)}$ and therefore $u \leq h$. Hence (5.1) implies that $u$ is moderate.

Let $D_{n}$ be a standard sequence approximating $E$ and let $\tau_{n}$ be the first exit time from $D_{n}$. It follows from (3.2) and Fatou's lemma that

$$
h(x) \leq \lim P_{x}\left\langle u, X_{D_{n}}\right\rangle .
$$

If $u$ is moderate and if it is dominated by an $L$-harmonic function $\tilde{h}$, then

$$
P_{x}\left\langle u, X_{D_{n}}\right\rangle \leq P_{x}\left\langle\tilde{h}, X_{D_{n}}\right\rangle=\Pi_{x} \tilde{h}\left(\xi_{\tau_{n}}\right) \leq \tilde{h}(x) .
$$

Hence $h<\infty$ and it is the minimal $L$-harmonic majorant of $u$.

Recall that, according to Section $4.2, H^{*}$ is the set of all $L$-harmonic functions $h$ of the form $h=u+\mathcal{E}(u)$ with positive $u$.

5.1.D. The class $H^{*}$ is a convex cone, and it contains with every $h$ all positive $L$-harmonic functions dominated by $h$.

Proof. By Lemma 1.2 in [8], to every $L$-harmonic function $h$ there corresponds a function $Z_{h}$ such that

$$
Z_{h}=\lim \left\langle h, X_{D_{n}}\right\rangle \quad \text { a.s. }
$$

for every standard sequence $D_{n}$ approximating $E$. Moreover,

$$
P_{x} Z_{h} \leq h(x) .
$$

By Theorem 1.5 in [8], (5.2) holds with the equality sign for $h \in H^{*}$. Lemma 5.3 in [10] in combination with Theorem 4.2 in [8] yields that, if (5.2) holds with the equality sign for an $L$-harmonic $h$, then $h \in H^{*}$. If this condition holds for $h_{1}, h_{2}$, then it holds for $c_{1} h_{1}+c_{2} h_{2}$ with positive constants $c_{1}, c_{2}$.

If $h_{1}, h_{2}$ are $L$-harmonic and if $h=h_{1}+h_{2} \in H^{*}$, then $P_{x} Z_{h}=h(x), P_{x} Z_{h_{1}} \leq$ $h_{1}(x)$ and $P_{x} Z_{h_{2}} \leq h_{2}(x)$. Since $Z_{h}=Z_{h_{1}}+Z_{h_{2}}$, we get $P_{x} Z_{h_{1}}=h_{1}(x)$ and therefore $h_{1} \in H^{*}$.

Remark. It follows from 5.1.D and Minkowski's inequality that the class $H^{* *}$ is a cone with the same properties as $H^{*}$.

Theorem 5.1. Let $\nu \in \mathcal{N}$. The following three conditions are equivalent:

(i) $\nu$ is finite;

(ii) the potential $h$ of $A^{\nu}$ is finite;

(iii) the log-potential $u$ of $A^{\nu}$ is a moderate solution.

Proof. The equivalence of (i) and (ii) is an implication of the relation

$$
h(x)=P_{x} A_{\infty}^{\nu}=\int k(x, y) \nu(d y),
$$

which follows from 4.3.B if $\nu \in \mathcal{N}^{* *}$ and which can be obtained by a monotone passage to the limit for an arbitrary $\nu \in \mathcal{N}$. The equivalence of (ii) and (iii) follows from 5.1.C.

For every closed subset $B$ of the Martin boundary $E^{\prime}$, we denote by $\mathcal{O}(B)$ the class of all open subsets $D$ of $E$ such that the $M$-closure of $D$ is disjoint from $B$. 
Theorem 5.2 (Extended mean value property). Let $u$ be a moderate solution with trace $\nu$. If $\nu$ is concentrated on a closed subset $B$ of $E^{\prime}$, then

$$
u(x)=-\log P_{x} e^{-\left\langle u, X_{D}\right\rangle} \quad \text { in } D
$$

for every $D \in \mathcal{O}(B)$.

The proof is based on

Lemma 5.1. Suppose $\tilde{D}_{m}=D_{m} \cap D$, where $D_{m}$ is a standard sequence approximating $E$ and $D \in \mathcal{O}(B)$. If $u \in \mathcal{U}$ is dominated by

$$
h_{B}(x)=\int_{B} k(x, y) \nu(d y)
$$

then, for every $\mu \in \mathcal{M}$,

$$
P_{\mu}\left\langle u 1_{D}, X_{\tilde{D}_{m}}\right\rangle \rightarrow 0 .
$$

Proof. Let $\tau$ and $\tau_{m}$ be the first exit times from $D$ and from $\tilde{D}_{m}$. By (1.13) and $(1.5)$,

$$
P_{\mu}\left\langle u 1_{D}, X_{\tilde{D}_{m}}\right\rangle=\Pi_{\mu}\left(u 1_{D}\right)\left(\xi_{\tau_{m}}\right) \leq \Pi_{\mu}\left(h_{B} 1_{D}\right)\left(\xi_{\tau_{m}}\right)=\Pi_{\mu}^{h_{B}}\left\{\xi_{\tau_{m}} \in D\right\} .
$$

Denote by $C$ the intersection of $E^{\prime}$ with the $M$-closure of $D$. Note that $C$ is disjoint from $B$ and $\left\{\xi_{\tau_{m}} \in D\right\} \downarrow\left\{\tau=\zeta, \xi_{\zeta-} \in C\right\}$. By (1.4),

$$
\Pi_{\mu}^{h_{B}}\left\{\xi_{\tau_{m}} \in D\right\} \rightarrow \Pi_{\mu}^{h_{B}}\left\{\tau=\zeta, \xi_{\zeta-} \in C\right\} \leq \int_{C \cap B} k(x, y) \nu(d y)=0 .
$$

Proof of Theorem 5.2. Let $D_{m}$ and $\tilde{D}_{m}$ be the sets introduced in Lemma 5.1. By the mean value property 2.1.D,

$$
u(x)=-\log P_{x} e^{-\left\langle u, X_{\tilde{D}_{m}}\right\rangle} \quad \text { in } \tilde{D}_{m} .
$$

Note that $\partial \tilde{D}_{m}=B_{m} \cup C_{m}$, where $B_{m}=\partial \tilde{D}_{m} \cap \partial D$, and $C_{m}=\partial \tilde{D}_{m} \cap D$ and therefore, for every $x \in \tilde{D}_{m}$,

$$
\left\langle u, X_{\tilde{D}_{m}}\right\rangle=\left\langle u 1_{B_{m}}, X_{\tilde{D}_{m}}\right\rangle+\left\langle u 1_{C_{m}}, X_{\tilde{D}_{m}}\right\rangle=\left\langle u, X_{m}\right\rangle+\left\langle u 1_{D}, X_{\tilde{D}_{m}}\right\rangle \quad P_{x^{-} \text {a.s. }},
$$

where $X_{m}$ is the restriction of $X_{\tilde{D}_{m}}$ to $\partial D$. By Lemma 5.1, the second term tends to 0 in $P_{\mu}$-probability as $m \rightarrow \infty$. Formula (5.3) will follow from (5.6) if we prove that, for every $x \in D$,

$$
\lim \left\langle u, X_{m}\right\rangle=\left\langle u, X_{D}\right\rangle \quad P_{x^{-}} \text {a.s. }
$$

By Lemma 3.1 of [9], $X_{m} \leq X_{m+1} \leq X_{D} P_{x}$-a.s., and therefore

$$
X_{m} \uparrow X^{*} \leq X_{D} \quad P_{x^{-} \text {a.s. }}
$$

By (1.13),

$$
\begin{aligned}
& P_{x}\left\langle 1, X_{m}\right\rangle=\Pi_{x}\left\{\xi_{\tau_{m}} \in \partial D\right\}, \\
& P_{x}\left\langle 1, X_{D}\right\rangle=\Pi_{x}\left\{\xi_{\tau} \in \partial D\right\},
\end{aligned}
$$

where $\tau_{m}, \tau$ are the first exit moments from $\tilde{D}_{m}, D$. Since $\left\{\xi_{\tau_{m}} \in \partial D\right\} \uparrow\left\{\xi_{\tau} \in \partial D\right\}$, (5.8) implies that $X^{*}=X_{D} P_{x}$-a.s. 
5.2. Operators $Q_{B}$. To extend the definition of the trace to all $u \in \mathcal{U}$, we construct a family of operators $Q_{B}: \mathcal{U} \rightarrow \mathcal{U}$.

Let $\mathcal{P}$ be a partially ordered set. We say that a sequence $p_{n} \in \mathcal{P}$ supports $\mathcal{P}$ if $p_{1} \prec p_{2} \prec \cdots \prec p_{n} \prec \ldots$ and if, for every $p \in \mathcal{P}$, there exists $N$ such that $p \prec p_{n}$ for all $n \geq N$. If $\varphi$ is a positive monotone increasing function on $\mathcal{P}$, then, for every sequence $\left\{p_{n}\right\}$ supporting $\mathcal{P}$, the $\operatorname{limit} \lim \varphi\left(p_{n}\right)$ exists and does not depend on the choice of $\left\{p_{n}\right\}$. We call it the limit of $\varphi$ along $\mathcal{P}$.

The set $\mathcal{O}(B)$ introduced in Section 5.1 is a partially ordered set relative to the inclusion. By Theorem 2.2, for every $u \in \mathcal{U}$ and every $x \in E, V_{D}(u)(x)$ is a monotone decreasing function on $\mathcal{O}(B)$, and therefore there exists a pointwise limit of $V_{D}(u)$ along $\mathcal{O}(B)$. We denote it $Q_{B}(u)$.

For every closed set $B \subset E^{\prime}$ and for every $\varepsilon>0$ we put

$$
D(B, \varepsilon)=\{x \in E: d(x, B)>\varepsilon\} .
$$

If $\varepsilon_{n} \downarrow 0$, then $D\left(B, \varepsilon_{n}\right)$ supports $\mathcal{O}(B)$. Clearly,

$$
Q_{B}(u)=\lim _{\varepsilon \rightarrow 0} V_{D(B, \varepsilon)}(u) .
$$

Note that a sequence $D_{n}$ supports $\mathcal{O}\left(E^{\prime}\right)$ if and only if it is a standard sequence approximating $E$.

Property 2.1.C implies that $Q_{B}(u) \in \mathcal{U}$. Operators $Q_{B}$ have the following properties:

5.2.A. $Q_{B}\left(u_{1}\right) \leq Q_{B}\left(u_{2}\right)$ for $u_{1} \leq u_{2}$.

5.2.B. $Q_{B}(u) \leq u$.

5.2.C. If $B_{1} \supset B_{2}$, then $Q_{B_{1}}(u) \geq Q_{B_{2}}(u)$.

5.2.D. For every $B_{1}, B_{2}, \quad Q_{B_{1} \cup B_{2}}(u) \leq Q_{B_{1}}(u)+Q_{B_{2}}(u)$.

5.2.E. $Q_{E^{\prime}}(u)=u$.

5.2.F. If $u \leq u_{1}+u_{2}$, then $Q_{B}(u) \leq Q_{B}\left(u_{1}\right)+Q_{B}\left(u_{2}\right)$.

Property 5.2.A follows from 2.3.A; 5.2.B follows from (2.17); 5.2.C follows from (2.18); 5.2.D is an implication of 2.3.D and the relation $D\left(B_{1} \cup B_{2}, \varepsilon\right)=D\left(B_{1}, \varepsilon\right) \cap$ $D\left(B_{2}, \varepsilon\right)$. 5.2.E holds because, by 2.1.D, $V_{D\left(E^{\prime}, \varepsilon\right)}(u)=u$ for all $\varepsilon$. Finally, 5.2.F follows from 2.3.C and 2.3.A.

5.2.G. For every moderate solution $u$, the trace of $u_{B}=Q_{B}(u)$ is concentrated on $B$.

Proof. If $\nu$ is the trace of $u$, then $u$ is dominated by $h$ defined by (1.1). Let $h_{B}$ be given by (5.4). By (1.4) and (1.5),

$$
h_{B}(x)=\Pi_{x}^{h}\left\{\xi_{\zeta-} \in B\right\}=\lim _{\varepsilon \rightarrow 0} \Pi_{x}^{h}\left\{\tau_{\varepsilon}<\zeta\right\}=\lim _{\varepsilon \rightarrow 0} \Pi_{x} h\left(\xi_{\tau_{\varepsilon}}\right),
$$

where $\tau_{\varepsilon}$ is the first exit time from $D(B, \varepsilon)$. By $(2.16)$,

$$
V_{D(B, \varepsilon)}(u)(x) \leq \Pi_{x} u\left(\xi_{\tau_{\varepsilon}}\right) \leq \Pi_{x} h\left(\xi_{\tau_{\varepsilon}}\right) .
$$

By (5.10), (5.11) and (5.12), $u_{B} \leq h_{B}$. Hence the trace of $u_{B}$ is concentrated on $B$.

5.2.H. If $u$ is a moderate solution with the trace concentrated on $B$, then

$$
Q_{B}(u)=u \text {. }
$$

This follows from Theorem 5.2 and (5.10).

5.2.I. If $Q_{B}(u)$ is moderate and if $B \subset B_{0}$, then $Q_{B_{0}}\left[Q_{B}(u)\right]=Q_{B}(u)=$ $Q_{B}\left[Q_{B_{0}}(u)\right]$. 
Indeed, by 5.2.G, the trace of $Q_{B}(u)$ is concentrated on $B$ and the first part of 5.2.I follows from 5.2.H. In particular, $Q_{B}\left[Q_{B}(u)\right]=Q_{B}(u)$. On the other hand, by 5.2.C and 5.2.A, $Q_{B}\left[Q_{B_{0}}(u)\right] \geq Q_{B}\left[Q_{B}(u)\right]=Q_{B}(u)$ and $Q_{B}\left[Q_{B_{0}}(u)\right] \leq Q_{B}(u)$ by 5.2.B and 5.2.A.

5.3. Stochastic boundary value on $B$. The following result can be proved by the same arguments as Theorem 3.1:

Theorem 5.3. For every $u \in \mathcal{U}$ and every closed subset $B$ of $E^{\prime}$, there exists a function $Z_{B}$ such that

$$
\lim \left\langle u, X_{D_{n}}\right\rangle=Z_{B} \quad P_{\mu} \text {-a.s. }
$$

for every sequence $D_{n}$ supporting $\mathcal{O}(B)$ and every $\mu \in \mathcal{M}$. For all $\mu \in \mathcal{M}$,

$$
P_{\mu} e^{-Z_{B}}=e^{-\left\langle Q_{B}(u), \mu\right\rangle} .
$$

In particular,

$$
Q_{B}(u)(x)=-\log P_{x} e^{-Z_{B}} .
$$

Corollary. For every $\mu \in \mathcal{M}$ and every $B$,

$$
\{\mathcal{R} \cap B=\emptyset\} \subset\left\{Z_{B}=0\right\} \quad P_{\mu} \text {-a.s. }
$$

If $B$ is $\mathcal{R}$-polar, then $Q_{B}(u)=0$.

Indeed, (5.17) follows from (5.14) and Lemma 4.1. If $B$ is $\mathcal{R}$-polar, then $Z_{B}=0$ $P_{\mu}$-a.s., and $Q_{B}(u)=0$ by $(5.16)$.

We call $Z_{B}$ a stochastic boundary value of the solution $u$ on set $B$.

\subsection{Expression of $Z_{B}$ in terms of $Q_{B}$.}

Theorem 5.4. Suppose that $u_{B}=Q_{B}(u)$ is a moderate solution. Then, for every standard sequence $D_{n}$ approximating $E$ and every $\mu \in \mathcal{M}$,

$$
Z_{B}=\lim \left\langle u_{B}, X_{D_{n}}\right\rangle \quad P_{\mu} \text {-a.s. }
$$

Proof. $1^{\circ}$. By Theorem 5.3, there exist functions $Z_{B}, \tilde{Z}_{B}$ and $\hat{Z}_{B}$ such that

$$
\begin{aligned}
& Z_{B}=\lim \left\langle u, X_{\tilde{D}_{n}}\right\rangle \quad P_{\mu} \text {-a.s. } \\
& \tilde{Z}_{B}=\lim \left\langle u_{B}, X_{\tilde{D}_{n}}\right\rangle \quad P_{\mu} \text {-a.s. }
\end{aligned}
$$

for every $\tilde{D}_{n}$ supporting $\mathcal{O}(B)$ and every $\mu$, and

$$
\hat{Z}_{B}=\lim \left\langle u_{B}, X_{D_{n}}\right\rangle \quad P_{\mu} \text {-a.s. }
$$

for every standard sequence $D_{n}$ approximating $E$ and every $\mu$. Moreover,

$$
P_{\mu} e^{-Z_{B}}=P_{\mu} e^{-\tilde{Z}_{B}}=P_{\mu} e^{-\hat{Z}_{B}}=e^{-\left\langle u_{B}, \mu\right\rangle}
$$

since $Q_{B}\left(u_{B}\right)=u_{B}$ by 5.2.I.

Formula (5.18) will follow from (5.22) if we show that $\hat{Z}_{B} \leq \tilde{Z}_{B} \leq Z_{B} P_{\mu}$-a.s. The second inequality follows from 5.2.B. It remains to prove that

$$
\hat{Z}_{B} \leq \tilde{Z}_{B} \quad P_{\mu} \text {-a.s. }
$$


$2^{\circ}$. Consider $D_{m n}=D_{m} \cap \tilde{D}_{n}$, where $D_{m}$ supports $\mathcal{O}\left(E^{\prime}\right)$ and $\tilde{D}_{n}$ supports $\mathcal{O}(B)$. By Lemma 5.1, $P_{\mu}\left\langle u_{B} 1_{\tilde{D}_{n}}, X_{D_{m n}}\right\rangle \rightarrow 0$ as $m \rightarrow \infty$, and therefore there exists a monotone increasing sequence $m_{n} \rightarrow \infty$ such that

$$
P_{\mu}\left\langle u_{B} 1_{\tilde{D}_{n}}, X_{D_{m_{n} n}}\right\rangle<1 / n \text {. }
$$

The sequence $D_{n}^{*}=D_{m_{n} n}$ supports $\mathcal{O}\left(E^{\prime}\right)$.

Note that $\partial D_{n}^{*}=B_{n} \cup C_{n}$, where $B_{n}=\partial D_{n}^{*} \cap \partial \tilde{D}_{n}$ and $C_{n}=\partial D_{n}^{*} \cap \tilde{D}_{n}$. By [9, Lemma 3.1], $X_{D_{n}^{*}} \leq X_{\tilde{D}_{n}}$ on $B_{n}$, and therefore

$$
\left\langle u_{B}, X_{D_{n}^{*}}\right\rangle \leq\left\langle u_{B} 1_{B_{n}}, X_{\tilde{D}_{n}}\right\rangle+\left\langle u_{B} 1_{C_{n}}, X_{D_{n}^{*}}\right\rangle \leq\left\langle u_{B}, X_{\tilde{D}_{n}}\right\rangle+\left\langle u_{B} 1_{\tilde{D}_{n}}, X_{D_{n}^{*}}\right\rangle .
$$

By (5.21), (5.20) and (5.24), this implies (5.18).

It follows from Theorem 5.3 and 5.2.C, D that:

5.4.A. If $B_{1} \supset B_{2}$, then $Z_{B_{1}} \geq Z_{B_{2}} P_{\mu}$-a.s. for all $\mu \in \mathcal{M}$.

5.4.B. For every $B_{1}, B_{2}, Z_{B_{1} \cup B_{2}} \leq Z_{B_{1}}+Z_{B_{2}} P_{\mu}$-a.s. for all $\mu \in \mathcal{M}$.

\subsection{Trace of $Q_{B}(u)$.}

Lemma 5.2. If $u$ is a moderate solution, then, for every $B, u_{B}=Q_{B}(u)$ is also moderate and the trace of $u_{B}$ is the restriction of the trace of $u$ to $B$. If the trace of $u$ does not charge $B$, then $u_{B}=0$.

Proof. Consider the minimal $L$-harmonic majorant

$$
h(x)=\int_{E^{*}} k(x, y) \nu(d y)
$$

of $u$ and the minimal $L$-harmonic majorant $h_{B}^{*}$ of $u_{B}$. By 5.2.G, $u_{B}$ is dominated by $h_{B}$ given by (5.4), and therefore

$$
h_{B}^{*} \leq h_{B} .
$$

By 5.2.B, there exists $\tilde{u}$ such that $\tilde{u}+\mathcal{E}(\tilde{u})=h_{B}$. The trace of $\tilde{u}$ is concentrated on $B$ and, by (5.13), $\tilde{u}_{B}=Q_{B}(\tilde{u})=\tilde{u}$. Hence $\tilde{h}_{B}=\tilde{u}_{B}+\mathcal{E}\left(\tilde{u}_{B}\right)=h_{B}$. By 5.1.A, $\tilde{u} \leq u$ because $h_{B} \leq h$. By 5.2.A, $\tilde{u}_{B} \leq u_{B}$ and therefore

$$
h_{B}=\tilde{h}_{B} \leq h_{B}^{*}
$$

By (5.25) and (5.26), $h_{B}^{*}=h_{B}$.

5.6. Properties of $w_{\Gamma}$. For every closed subset $\Gamma$ of $E^{\prime}$ we put

$$
w_{\Gamma}(x)=-\log P_{x}\{\mathcal{R} \cap \Gamma=\emptyset\} .
$$

5.6.A. We have

$$
Q_{\Gamma}\left(w_{\Gamma}\right)=w_{\Gamma} .
$$

Proof. By Theorem 8.2 in [5], for every measure $\mu$ with support disjoint from $\Gamma$,

$$
P_{\mu}\{\mathcal{R} \cap \Gamma=\emptyset\}=e^{-\left\langle w_{\Gamma}, \mu\right\rangle} .
$$

Let $D_{n}$ be a supporting sequence of $\mathcal{O}(\Gamma)$. By the definition of $Q_{\Gamma},(2.15)$ and (5.29),

$$
Q_{\Gamma}\left(w_{\Gamma}\right)=-\lim \log P_{x} P_{X_{D_{n}}}\{\mathcal{R} \cap \Gamma=\emptyset\} .
$$

By (4.11), $\{\mathcal{R} \cap \Gamma=\emptyset\}$ belongs to the $P_{x}$-completion of $\mathcal{F}_{\supset D_{n}}$; and, by (1.12),

$$
P_{x} P_{X_{D_{n}}}\{\mathcal{R} \cap \Gamma=\emptyset\}=P_{x}\{\mathcal{R} \cap \Gamma=\emptyset\}=e^{-w_{\Gamma}(x)} .
$$


5.6.B. If $w_{\Gamma}$ is a moderate solution, then $\Gamma$ is $\mathcal{R}$-polar.

Indeed, $w_{\Gamma}$ is the log-potential of $Z(\Gamma)$ given by (4.12). By 5.1.C, $h(x)=$ $P_{x} Z(\Gamma)<\infty$, which implies 5.6.B.

5.6.C. For every $u \in \mathcal{U}$ and every closed $B \subset \Gamma$,

$$
Q_{B}(u) \leq w_{\Gamma} .
$$

Indeed, by (5.16) and (5.17),

$$
Q_{\Gamma}(u)(x)=-\log P_{x} e^{-Z_{\Gamma}} \leq-\log P_{x}\left\{Z_{\Gamma}=0\right\} \leq-\log P_{x}\{\mathcal{R} \cap \Gamma=\emptyset\} .
$$

(5.30) follows from (5.31) by 5.2.C.

5.6.D. If the spectral measure $\nu$ of a CLA $A$ is concentrated on $\Gamma$ and if $u$ is the $\log$-potential of $A$, then

$$
u=Q_{\Gamma}(u) \leq w_{\Gamma} .
$$

Proof. Note that, if $u_{n}=Q_{\Gamma}\left(u_{n}\right)$ and if $u_{n} \uparrow u$, then $Q_{\Gamma}(u)=u$ by 5.2.A and 5.2.B. Therefore it is sufficient to prove (5.32) for $\nu \in \mathcal{N}^{* *}$. By Theorem 5.1, in this case $u$ is moderate. By Lemma $5.2, u_{\Gamma}=Q_{\Gamma}(u)$ is a moderate solution with the same trace as $u$, and therefore $u_{\Gamma}=u$. The second part of (5.32) follows from 5.6.A.

5.6.E. If $\nu$ is a finite measure concentrated on $\Gamma$ and if $u$ is the log-potential of $A^{\nu}$, then $A_{\infty}^{\nu}$ is $\mathcal{M}^{*}$-equivalent to the stochastic boundary value $Z_{\Gamma}$ of $u$ on $\Gamma$.

Indeed, let $\mu \in \mathcal{M}^{*}$ and let $D_{n}$ be a standard sequence approximating $E$. By Theorems 4.4 and 3.2,

$$
A_{\infty}^{\nu}=\lim \left\langle u, X_{D_{n}}\right\rangle \quad P_{\mu} \text {-a.s. }
$$

By Theorem 5.3,

$$
Z_{\Gamma}=\lim \left\langle u_{\Gamma}, X_{D_{n}}\right\rangle \quad P_{\mu} \text {-a.s., }
$$

and $P_{\mu}\left\{A_{\infty}^{\nu}=Z_{\Gamma}\right\}=1$ by 5.6.D.

5.7. General definition of the trace. Fix a solution $u$. We say that a compact set $B \subset E^{\prime}$ is moderate for $u$ if the solution $u_{B}=Q_{B}(u)$ is moderate. Let $\nu_{B}$ stand for the trace of $u_{B}$. By 5.2.D, the union of two moderate sets is moderate. Suppose that $B$ is moderate, and let $\tilde{B} \subset B$. By Lemma 5.2, $\tilde{B}$ is moderate. By 5.2.I, $Q_{B} Q_{\tilde{B}}(u)=Q_{\tilde{B}}(u)$, and, by Lemma $5.2, \nu_{\tilde{B}}$ is the restriction of $\nu_{B}$ to $\tilde{B}$.

A relatively open subset $A$ of $E^{\prime}$ is called moderate if all compact subsets of $A$ are moderate. The union $O$ of all moderate open sets is moderate. Clearly, there exists a unique measure $\nu$ on $O$ such that its restriction to an arbitrary compact subset $B$ coincides with $\nu_{B}$. This measure is a Radon measure on $O$ (that is, it is finite on all compact subsets). By 5.1.B, $\nu(B)=0$ for all $\mathcal{R}$-polar sets $B$. We call closed set $\Gamma=E^{\prime} \backslash O$ the singular set of the solution $u$, and we call the pair $(\Gamma, \nu)$ the trace of $u$ on $E^{\prime}$.

\section{Trace of $(\Gamma, \nu)$-Solutions}

6.1. Normal pairs. According to the definition in Section 5.7, the trace of every $u \in \mathcal{U}$ is a pair $(\Gamma, \nu)$ with the following properties:

6.1.A. $\Gamma$ is a closed subset of $E^{\prime}$.

6.1.B. $\nu$ is a Radon measure on $\mathcal{O}=E^{\prime} \backslash \Gamma$.

6.1.C. $\nu$ does not charge $\mathcal{R}$-polar sets. 
We say that $x$ is an explosion point of a measure $\nu$ if $\nu(U)=\infty$ for every neighborhood $U$ of $x$. If no explosion point belongs to a compact set $B$, then $\nu(B)<\infty$. Condition 6.1.B is equivalent to:

6.1.B'. $\mathcal{O}$ contains no explosion points of $\nu$.

For every $(\Gamma, \nu)$ subject to conditions 6.1.A,B,C, we denote by $\mathcal{L}(\Gamma, \nu)$ the class of all $\mathcal{R}$-polar relatively open subsets of $\Gamma$ which contain no explosion points of $\nu$. Note that if $\Lambda=\Gamma \backslash \Gamma_{0} \in \mathcal{L}(\Gamma, \nu)$, then $\{\mathcal{R} \cap \Gamma=\emptyset\}=\left\{\mathcal{R} \cap \Gamma_{0}=\emptyset\right\} P_{x}$-a.s. for all $x \in E$, and therefore a $(\Gamma, \nu)$-solution is at the same time a $\left(\Gamma_{0}, \nu\right)$-solution. We say that $(\Gamma, \nu)$ is a normal pair if the empty set is the only element of $\mathcal{L}(\Gamma, \nu)$.

Theorem 6.1. Every $(\Gamma, \nu)$-solution $u$ is also a $\left(\Gamma_{0}, \nu\right)$-solution with a normal pair $\left(\Gamma_{0}, \nu\right)$.

Proof. The union of an arbitrary family of $\mathcal{R}$-polar sets relatively open in $\Gamma$ is $\mathcal{R}$ polar. Therefore the union $\Lambda_{0}$ of all $\Lambda \in \mathcal{L}(\Gamma, \nu)$ belongs to $\mathcal{L}(\Gamma, \nu)$ and $u$ is a $\left(\Gamma_{0}, \nu\right)$-solution. Put $\Gamma_{0}=\Gamma \backslash \Lambda_{0}$. Let $\Lambda_{1} \in \mathcal{L}\left(\Gamma_{0}, \nu\right)$. Then $\Gamma_{1}=\Gamma_{0} \backslash \Lambda_{1}$ is closed and $\Lambda^{*}=\Gamma \backslash \Gamma_{1}=\Lambda_{0} \cup \Lambda_{1}$ belongs to $\mathcal{L}(\Gamma, \nu)$. Hence $\Lambda^{*} \subset \Lambda_{0}$, which implies $\Lambda_{1} \subset \Lambda_{0}$. On the other hand, $\Lambda_{1} \subset \Gamma_{0}=\Gamma \backslash \Lambda_{0}$. Hence $\Lambda_{1}=\emptyset$, and the pair $\left(\Gamma_{0}, \nu\right)$ is normal.

6.2. It follows from [12, Theorem 3.1] and 5.2.A that the following condition holds if $E$ is a bounded domain in $\mathbb{R}^{d}$ with the smooth boundary and if $L$ is a second order elliptic operator in $\mathbb{R}^{d}$ :

$(\mathbb{N}) Q_{B}\left[Q_{\Gamma}(u)\right]=0$ for every $u \in \mathcal{U}$ and any disjoint closed subsets $B, \Gamma$ of $E^{\prime}$.

In Section 8 we prove a more general result which implies, in particular, that condition $(\mathbb{N})$ holds, if $E$ is a bounded domain in $\mathbb{R}^{d}, L$ is a second order elliptic differential operator in $E$ with bounded coefficients, and distinct points of the Martin boundary $E^{\prime}$ correspond to distinct points of the Euclidean boundary $\partial E$.

Condition $(\mathbb{N})$ is equivalent to

$\left(\mathbb{N}^{\prime}\right) Q_{B}\left(W_{\Gamma}\right)=0$ for any disjoint closed sets $B, \Gamma$.

Indeed, $(\mathbb{N})$ implies $\left(\mathbb{N}^{\prime}\right)$ by 5.6.A, and $\left(\mathbb{N}^{\prime}\right)$ implies $(\mathbb{N})$ by 5.6.C and 5.2.A.

In the rest of Section 6 and in Section 7 we consider only superdiffusions which satisfy condition $(\mathbb{N})$. Here are two immediate implication of this condition:

6.2.A. If $u$ is the $\log$-potential of $A^{\nu}$ and if $\nu$ is concentrated on $\Gamma$, then

$$
Q_{B}(u)=0
$$

for every $B$ disjoint from $\Gamma$.

This follows from 5.6.D and $\left(\mathbb{N}^{\prime}\right)$.

6.2.B. Let $u$ be the log-potential of $A^{\nu}$. If $\nu(B)=0$ and if $\nu(U)<\infty$ for a neighborhood $U$ of $B$, then $Q_{B}(u)=0$.

Proof. Denote by $\nu_{1}, \nu_{2}$ the restrictions of $\nu$ to $U$ and to $E^{\prime} \backslash U$. Let $u_{1}$ and $u_{2}$ be the $\log$-potentials of $A^{\nu_{1}}$ and $A^{\nu_{2}}$. By Lemma 3.1, $u \leq u_{1}+u_{2}$, and, by 5.2.F, $Q_{B}(u) \leq Q_{B}\left(u_{1}\right)+Q_{B}\left(u_{2}\right)$.

By Theorem 5.1, $u_{1}$ is a moderate solution. By Lemma 5.2, $Q_{B}\left(u_{1}\right)=0$.

By 5.6.D,$u_{2} \leq w_{\Gamma}$, where $\Gamma=E^{\prime} \backslash U$. Since $B$ and $\Gamma$ are disjoint, $Q_{B}\left(u_{2}\right) \leq$ $Q_{B}\left(w_{\Gamma}\right)=0$ by $5.2 . \mathrm{A}$ and $(\mathbb{N})$.

Lemma 6.1. Let $u$ be the $(\Gamma, \nu)$-solution and let $B$ be a closed subset of $O=E^{\prime} \backslash \Gamma$. Then the trace of $Q_{B}(u)$ is equal to the restriction $\nu^{\prime}$ of $\nu$ to $B$. 
Proof. Let $A^{\prime}=A_{\infty}^{\nu^{\prime}}$ and $A^{\prime \prime}=A_{\infty}^{\nu^{\prime \prime}}$, where $\nu^{\prime \prime}$ is the restriction of $\nu$ to $B^{\prime \prime}=O \backslash B$. Denote by $u^{\prime}, u^{\prime \prime}$ the log-potentials of $A^{\prime}, A^{\prime \prime}$. By (4.9), $u^{\prime} \leq u$, and therefore $u^{\prime}=Q_{B}\left(u^{\prime}\right) \leq Q_{B}(u)$ by 5.2.A and 5.6.D. On the other hand, $u$ is the log potential of $Z=A^{\prime}+A^{\prime \prime}+Z(\Gamma)$, where $Z(\Gamma)$ is given by (4.12). Therefore

$$
Q_{B}(u) \leq Q_{B}\left(u^{\prime}\right)+Q_{B}\left(u^{\prime \prime}\right)+Q_{B}\left(w_{\Gamma}\right)
$$

by Lemma 3.1 and 5.2.F. It remains to note that $Q_{B}\left(u^{\prime \prime}\right)=0$ by 6.2 .B and $Q_{B}\left(w_{\Gamma}\right)=0$ by $(\mathbb{N})$. This yields $Q_{B}(u)=u^{\prime}$.

\subsection{Trace of a $(\Gamma, \nu)$-solution.}

Theorem 6.2. Let $(\Gamma, \nu)$ be a normal pair. If $u$ is a $(\Gamma, \nu)$-solution, then the trace of $u$ is equal to $(\Gamma, \nu)$.

Proof. Denote the trace of $u$ by $\left(\Gamma_{0}, \nu_{0}\right)$. By the definition of the trace (see Section 5.7), $\mathcal{O}_{0}=E^{\prime} \backslash \Gamma_{0}$ is the maximal moderate open set, and therefore $\mathcal{O} \subset \mathcal{O}_{0}$ by Lemma 6.1 .

If $B$ is a closed subset of $\mathcal{O}$, then $B \subset \mathcal{O}_{0}$, and the trace of $Q_{B}(u)$ is equal to the restriction of $\nu_{0}$ by the definition of $\nu_{0}$. Therefore $\nu=\nu_{0}$ on $\mathcal{O}$. Theorem 6.2 will be proved if we show that $\Lambda=\Gamma \backslash \Gamma_{0}$ belongs to $\mathcal{L}(\Gamma, \nu)$. $\Lambda$ contains no explosion points of $\nu$. Indeed, since $\nu$ is concentrated on $\mathcal{O}$, every explosion point of $\nu$ is also an explosion point for $\nu_{0}$ and, by the definition of the trace, it belongs to $\Gamma_{0}$. It remains to check that $\Lambda$ is $\mathcal{R}$-polar. If $B$ is a closed subset of $\Lambda$, then $B \subset \mathcal{O}_{0}$ and therefore it is moderate for $u$. By 5.2.A and (5.28), $Q_{B}(u) \geq Q_{B}\left(w_{B}\right)=w_{B}$. Hence, $B$ is moderate. By Theorem 5.4, it is $\mathcal{R}$-polar.

\subsection{Trace of an arbitrary solution.}

Theorem 6.3. The trace $(\Gamma, \nu)$ of an arbitrary solution $u$ is a normal pair.

First we prove two lemmas.

Lemma 6.2. If $D_{1}, D_{2}, \tilde{D}$ are open subsets of $E$ and if

$$
D_{1} \cap \tilde{D}=D_{2} \cap \tilde{D}
$$

then

$$
\{\mathcal{R} \subset \tilde{D}\} \subset\left\{X_{\tilde{D}}=0\right\} \subset\left\{X_{D_{1}}=X_{D_{2}}\right\} \quad P_{x} \text {-a.s. }
$$

Proof. Let $U_{n}$ be a standard sequence approximating $\tilde{D}$. By [3, Lemma 2.1], $\{\mathcal{R} \subset$ $\left.U_{n}\right\} \subset\left\{X_{\tilde{D}}=0\right\}$ a.s., which implies the first part of (6.3).

A set $Q=\left\{X_{\tilde{D}}=0, X_{D_{1}} \neq X_{D_{2}}\right\}$ belongs to $\mathcal{F}_{\supset U}$, where $U=\tilde{D} \cap D_{1}=\tilde{D} \cap D_{2}$. By (1.12), $P_{x}(Q)=P_{x} P_{X_{U}}(Q)$ and, by [5, II.4.6], the second part of (6.3) will be proved if we show that $P_{x}(Q)=0$ for all $x \notin U$. We have $P_{x}\left\{X_{\tilde{D}}=\delta_{x}\right\}=1$ if $x \notin \tilde{D}$, and $P_{x}\left\{X_{D_{1}}=X_{D_{2}}=\delta_{x}\right\}=1$ if $x \in \tilde{D} \cap U^{c} \subset\left(D_{1} \cup D_{2}\right)^{c}$.

Lemma 6.3. If $(\Gamma, \nu)$ is the trace of $u$ and if $\Gamma$ is $\mathcal{R}$-polar and $\nu$ is finite, then $u$ is moderate.

Remark. According to the definition of the trace in Section 5.7, if $u$ is moderate, then $\Gamma$ is empty. 
Proof. Let $Z$ be the stochastic boundary value of $u$ and let $Z_{B}$ be its stochastic boundary value on $B$. Put $B_{n}=\left\{x \in E^{\prime}: d(x, \Gamma) \geq 1 / n\right\}$. Note that $B_{n} \uparrow O=$ $E^{\prime} \backslash \Gamma$. By (3.4) and (5.16),

$$
\begin{aligned}
u(x) & =-\log P_{x} \exp \{-Z\}, \\
u_{n}(x)=Q_{B_{n}}(x) & =-\log P_{x} \exp \left\{-Z_{B_{n}}\right\} .
\end{aligned}
$$

The trace of $u_{n}$ is the restriction of $\nu$ to $B_{n}$, and therefore

$$
u_{n}(x)+\mathcal{E}\left(u_{n}\right)(x)=\int_{B_{n}} k(x, y) \nu(d y)<\infty .
$$

Hence, for all $x$ and $n$,

$$
u_{n}(x) \leq h(x)=\int_{O} k(x, y) \nu(d y) .
$$

By 5.4.B, there exists $\tilde{Z}$ such that $Z_{B_{n}} \uparrow \tilde{Z} P_{x}$-a.s. for all $x$. We have

$$
-\log P_{x} e^{-\tilde{Z}}=\lim u_{n}(x) \leq h(x) .
$$

To prove that $u$ is moderate, it is sufficient to show that, for every $x$,

$$
\tilde{Z}=Z \quad P_{x} \text {-a.s. }
$$

We apply Lemma 6.2 to $D_{1}=D\left(B_{n}, \varepsilon\right), D_{2}=D\left(E^{\prime}, \varepsilon\right), \tilde{D}=D(\Gamma, \delta)$. Condition (6.2) holds if $\varepsilon<\delta$ and $d(x, \Gamma)<\delta-\varepsilon$ for all $x \notin B_{n}$. We conclude from (6.3) that

$$
P_{x}\left\{\mathcal{R} \subset D(\Gamma, \delta), \exp \left[-\left\langle u, X_{D\left(B_{n}, \varepsilon\right)}\right\rangle\right]\right\}=P_{x}\left\{\mathcal{R} \subset D(\Gamma, \delta), \exp \left[-\left\langle u, X_{D\left(E^{\prime}, \varepsilon\right)}\right\rangle\right]\right\} .
$$

By passing to the limit, first as $\varepsilon \rightarrow 0$, then as $n \rightarrow \infty$, and finally as $\delta \rightarrow 0$, we get

$$
P_{x}\left\{\mathcal{R} \cap \Gamma=\emptyset, e^{-\tilde{Z}}\right\}=P_{x}\left\{\mathcal{R} \cap \Gamma=\emptyset, e^{-Z}\right\}
$$

Since $\Gamma$ is $\mathcal{R}$-polar and since $P_{x}\{\tilde{Z} \leq Z\}=1$ by 5.4 .A, we get (6.4).

Proof of Theorem 6.3. $1^{\circ}$. Let $\Lambda \in \mathcal{L}(\Gamma, \nu)$ and let $\Gamma_{0}=\Gamma \backslash \Lambda$. The theorem will be proved if we show that $v=Q_{B_{0}}(u)$ is moderate for every closed subset $B_{0}$ of $O_{0}=E^{\prime} \backslash \Gamma_{0}$. Indeed, this implies $O_{0} \subset O$ and therefore $\Gamma_{0} \supset \Gamma, \Lambda=\emptyset$.

Let $\left(\Gamma_{1}, \nu_{1}\right)$ be the trace of $v$. By Lemma 6.3 , it is sufficient to to prove that $\Gamma_{1}$ is $\mathcal{R}$-polar and $\nu_{1}$ is finite.

$2^{\circ}$. By 5.2.B, $v \leq u$, and by 5.2.A, all moderate sets for $u$ are also moderate for $v$. Hence $\Gamma_{1} \subset \Gamma$. By $(\mathbb{N}), Q_{B}\left[Q_{B_{0}}\right](u)=0$ for all $B \subset E^{\prime} \backslash B_{0}$. Hence, $E^{\prime} \backslash B_{0}$ is moderate for $v$ and is contained in $O_{1}$. We conclude that $\Gamma_{1} \subset B_{0} \cap \Gamma$, which is a subset of $\mathcal{R}$-polar set $\Lambda$.

$3^{\circ}$. Note that $B_{0} \subset O \cup \Lambda$ does not contain explosion points of $\nu$, and therefore $\nu\left(B_{0}\right)<\infty$. Since $Q_{B}(v)=0$ for $B \cap B_{0}=\emptyset$, the measure $\nu_{1}$ vanishes on $E^{\prime} \backslash B_{0}$. Since $v \leq u, \nu_{1} \leq \nu$ on $O \subset O_{1}$. We have

$$
\nu_{1}\left(O_{1}\right)=\nu_{1}\left(O_{1} \cap B_{0}\right)=\nu_{1}\left[\left(O_{1} \backslash O\right) \cap B_{0}\right]+\nu_{1}\left(O \cap B_{0}\right) \leq \nu\left(O \cap B_{0}\right)<\infty,
$$

because $\nu_{1}\left(\Gamma \cap B_{0}\right)=0$ by 5.1 . B. 


\section{MAXimal PROPERTY of $(\Gamma, \nu)$-SOlutions}

7.1. Our objective is to prove:

Theorem 7.1. The $(\Gamma, \nu)$-solution corresponding to a normal pair $(\Gamma, \nu)$ dominates all solutions $u$ with the same trace.

This follows easily from a kind of mean value theorem which is also of independent interest:

Theorem 7.2. Let $u$ be a solution with the trace $(\Gamma, \nu)$. For every $D \in \mathcal{O}(\Gamma)$, there exists a positive function $Z^{*}(D)$ such that

$$
Z^{*}(D) \leq A_{\infty}^{\nu} \quad \text { a.s. }
$$

and

$$
u(x)=-\log P_{x} e^{-Z^{*}(D)-\left\langle u, X_{D}\right\rangle} \quad \text { in } D .
$$

To prove Theorem 7.1, we apply Theorem 7.2 to domains $D_{n}=D(\Gamma, 1 / n)$. Formulae (7.1) and (7.2) imply that

$$
u(x) \leq-\log P_{x} e^{-A^{\nu}} e^{-\left\langle u, X_{D_{n}}\right\rangle}
$$

for every $n$. It follows from (5.14) and (5.17) that

$$
u(x) \leq-\log P_{x} e^{-A^{\nu}} e^{-Z_{\Gamma}} \leq-\log P_{x}\left\{Z_{\Gamma}=0, e^{-A^{\nu}}\right\} \leq-\log \left\{\mathcal{R} \cap \Gamma=\emptyset, e^{-A_{\infty}^{\nu}}\right\}
$$

which proves Theorem 7.1.

7.2. Proof of Theorem 7.2. $1^{\circ}$. Denote by $B$ the intersection of $E^{\prime}$ with the $M$-closure of $D$. Put $\tilde{D}_{n}=D \cap D_{n}$, where $D_{n}=D(B, 1 / n)$. By the mean value property 2.1.D,

$$
u(x)=-\log P_{x} e^{-\left\langle u, X_{\tilde{D}_{n}}\right\rangle} \quad \text { in } \tilde{D}_{n} .
$$

For every $x \in \tilde{D}_{n}$, the measure $X_{\tilde{D}_{n}}$ is concentrated, $P_{x}$-a.s., on $\bar{D}=\partial D \cup D$, and therefore

$$
\left\langle u, X_{\tilde{D}_{n}}\right\rangle=\left\langle u_{\partial D}, X_{\tilde{D}_{n}}\right\rangle+\left\langle u_{D}, X_{\tilde{D}_{n}}\right\rangle \quad P_{x^{-} \text {-a.s. }}
$$

Formula (7.2) will follow from (7.4) if we prove that, for every $x \in D$,

$$
\lim \left\langle u_{\partial D}, X_{\tilde{D}_{n}}\right\rangle=\left\langle u, X_{D}\right\rangle \quad P_{x^{-} \text {a.s. }}
$$

and

$$
\left\langle u_{D}, X_{\tilde{D}_{n}}\right\rangle \rightarrow Z^{*}(D) \quad P_{x} \text {-a.s. }
$$

$2^{\circ}$. Note that $\left\langle u_{\partial D}, X_{\tilde{D}_{n}}\right\rangle=\left\langle u, X_{n}\right\rangle$, where $X_{n}$ is the restriction of the measure $X_{\tilde{D}_{n}}$ to $\partial D$. Therefore we get (7.5) if we prove that $X_{n} \uparrow X_{D} P_{x^{-}}$-a.s. for $x \in D$. This has been already done at the end of the proof of Theorem 5.2.

$3^{\circ}$. Denote by $\bar{X}_{n}$ the restriction of $X_{\tilde{D}_{n}}$ to $D$. By (1.12), (1.8) and 2.1.D,

$$
\begin{aligned}
P_{x}\left\{e^{-\left\langle u_{D}, X_{\tilde{D}_{n+1}}\right\rangle} \mid \mathcal{F}_{\subset \tilde{D}_{n}}\right\} & =P_{{X_{\tilde{D}}}_{n}} e^{-\left\langle u_{D}, X_{\tilde{D}_{n+1}}\right\rangle} \\
& \geq P_{\bar{X}_{n}} e^{-\left\langle u_{D}, X_{\tilde{D}_{n+1}}\right\rangle}=e^{-\left\langle u, \bar{X}_{n}\right\rangle}=e^{-\left\langle u_{D}, X_{\tilde{D}_{n}}\right\rangle} .
\end{aligned}
$$

Therefore $e^{-\left\langle u_{D}, X_{\tilde{D}_{n}}\right\rangle}$ is a bounded submartingale relative to $P_{x}$ (cf. the proof of Theorem 3.1), and there exists $Z^{*}(D)$ subject to (7.6). 
To get (7.1), we recall that, by Section 3.5, the restriction $\nu_{B}$ of $\nu$ to $B$ is the trace of $Q_{B}(u)$. By Theorem 5.3 and 5.6.E, the corresponding functional $A^{\nu_{B}}$ satisfies the condition

$$
A^{\nu_{B}}=\lim \left\langle u, X_{D_{n}}\right\rangle \quad \text { a.s. }
$$

Lemma 3.1 in [9] implies that

$$
\left\langle u_{D}, X_{\tilde{D}_{n}}\right\rangle \leq\left\langle u_{D}, X_{D_{n}}\right\rangle \leq\left\langle u, X_{D_{n}}\right\rangle .
$$

Hence,

$$
Z^{*}(D)=\lim \left\langle u_{D}, X_{\tilde{D}_{n}}\right\rangle \leq A^{\nu_{B}} \leq A^{\nu} \quad \text { a.s. }
$$

\section{ON CONDITION $(\mathbb{N})$}

8.1. In this section we introduce a concept of a normal point of the Martin boundary $E^{\prime}$ and we prove that condition $(\mathbb{N})$ holds if all points of $E^{\prime}$ are normal. In the second part of the section we establish tests of normality.

For every $c \in E^{\prime}$, we put $V_{\varepsilon}(c)=\{x \in E: d(x, c)<\varepsilon\}$. More generally, $V_{\varepsilon}(B)=\{x \in E: d(x, B)<\varepsilon\}$ for every $B \subset E^{\prime}$.

8.2. Admissible pairs and normal points. Let $c \in E^{\prime}$ and let $D$ be an open subset of $E$. We say that $(c, D)$ is an admissible pair if there exist $\delta>0$ and $\varepsilon>0$ such that $V_{\delta}(c) \subset D$ and

$$
P_{x}\left\{X_{D}=0\right\} \geq \varepsilon \quad \text { for all } x \in V_{\delta}(c) .
$$

Note that, if $(c, D)$ is admissible, then $(c, \tilde{D})$ is admissible for every $\tilde{D} \supset D$. Indeed, by (1.12),

$$
P_{x}\left\{X_{\tilde{D}}=0\right\}=P_{x} P_{X_{D}}\left\{X_{\tilde{D}}=0\right\} \geq P_{x}\left\{X_{D}=0\right\} .
$$

A point $c \in E^{\prime}$ is called normal if, for every $\varepsilon>0$, there exists an admissible pair $(c, D)$ such that $D \subset V_{\varepsilon}(c)$. Our first objective is to prove

Theorem 8.1. Condition $(\mathbb{N})$ holds if all points of $E^{\prime}$ are normal.

8.3. The proof needs some preparations. With every open subset $D$ of $E$ we associate a relatively open subset $D^{*}$ of $E^{\prime}$ defined by the condition: $c \in D^{*}$ if $V_{\varepsilon}(c) \subset D$ for some $\varepsilon>0$. Note that $\hat{D}=D \cup D^{*}$ is an open set in the exit space $\hat{E}$. Recall that to every function $f$ on $E$ there corresponds a random variable $f^{E}$ defined by formula (2.3).

Lemma 8.1. Suppose that $\tau$ is the first exit time from an open set $D \subset E, \varphi$ is a positive bounded real-valued function on $\partial D$ and

$$
f(x)=\Pi_{x} \varphi\left(\xi_{\tau}\right) .
$$

Then

$$
f^{E}=0 \quad \text { a.s. on }\left\{\xi_{\zeta-} \in D^{*}\right\} .
$$

Proof. It is sufficient to prove that

$$
\Pi_{x}\left\{\xi_{\zeta-} \in D_{0}^{*}, f^{E}\right\}=0
$$

for every $x \in E$ and every open set $D_{0}$ such that $d\left(D_{0}, E \backslash D\right)>0$. Consider stopping times $\sigma_{n}, \tau_{n}$ defined as follows: $\tau_{1}=\tau$;

$$
\sigma_{n}=\inf \left\{t: t>\tau_{n}, \xi_{t} \in D_{0}\right\}
$$


if $\xi_{t} \in D_{0}$ for some $t>\tau_{n} ; \sigma_{n}=\zeta$ otherwise;

$$
\tau_{n+1}=\inf \left\{t: t>\sigma_{n}, \xi_{t} \in E \backslash D\right\}
$$

if $\xi_{t} \in E \backslash D$ for some $t>\sigma_{n}, \tau_{n+1}=\zeta$ otherwise. Put $A_{n}=\left\{\tau_{1}<\sigma_{1}<\cdots<\right.$ $\left.\sigma_{n}<\tau_{n}=\zeta\right\}$. By the strong Markov property,

$$
\Pi_{x}\left\{A_{n}, f^{E}\right\}=\Pi_{x}\left[\tau_{1}<\sigma_{1}<\cdots<\sigma_{n}, \Pi_{\xi_{\sigma_{n}}}\left\{\tau=\zeta, f^{E}\right\}\right] .
$$

By Lemma 2.1, $\Pi_{y}\left\{\tau=\zeta, f^{E}\right\}=\Pi_{y}\left\{\tau=\zeta, f^{D}\right\}$ is equal to 0 for all $y$, and therefore $\Pi_{x}\left\{A_{n}, f^{E}\right\}=0$. Since the union of the $A_{n}$ coincides with $\left\{\xi_{\zeta-} \in D_{0}^{*}\right\}$, this implies $(8.3)$.

Lemma 8.2. If

$$
v(x)=-\log P_{x}\left\{X_{D}=0\right\}
$$

and if all points of $D^{*}$ are normal, then for every $x \in E$,

$$
v^{E}=0 \quad P_{x} \text {-a.s. on }\left\{\xi_{\zeta-} \in D^{*}\right\} \text {. }
$$

For every closed set $B \subset D^{*}$, there exists $\delta>0$ such that $v$ is bounded in $V_{\delta}(B)$.

Proof. For each $c \in B$, there exists $\varepsilon(c)>0$ such that $v$ is bounded on $V[c]=$ $V_{\varepsilon(c)}(c)$. The sets $\hat{V}[c]$ form an open cover of $B$. Let $\hat{V}\left[c_{1}\right], \ldots, \hat{V}\left[c_{n}\right]$ be a finite subcover and let $D_{1}$ be the union of the $V\left[c_{i}\right], i=1, \ldots, n$. If $\delta>0$ is sufficiently small, then $V_{\delta}(B) \subset D_{1}$, which proves the second part of the lemma.

To prove the first part, we apply Lemma 8.1 to $D_{0}=V_{\delta / 2}(B)$ and $\varphi=v$. By the Markov property (1.12),

$$
v(x)=-\log P_{x} e^{-\left\langle v, X_{D_{0}}\right\rangle},
$$

and, by Jensen's inequality, $v \leq f$, where $f(x)=P_{x}\left\langle v, X_{D_{0}}\right\rangle$. By (1.13), $f(x)=$ $\Pi_{x} v\left(\xi_{\tau_{0}}\right)$, where $\tau_{0}$ is the first exit time from $D_{0}$. By Lemma 8.1, $f^{E}=0$ a.s. on $\left\{\xi_{\zeta-} \in D_{0}^{*}\right\}$, which implies an analogous relation for $v$. Since $B$ is an arbitrary closed subset of $D^{*}$, we get (8.5).

Lemma 8.3. Let $\tilde{u}=Q_{\Gamma}(u)$. If all points of $E^{\prime} \backslash \Gamma$ are normal, then, for every $\delta>0$,

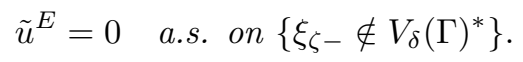

For every closed set $B$ disjoint from $\Gamma$, there exists $\delta>0$ such that $\tilde{u}$ is bounded in $V_{\delta}(B)$.

Proof. If $B \subset E^{\prime} \backslash \Gamma$, then there is a $D \in \mathcal{O}(\Gamma)$ such that $B \subset D^{*}$. Since $1_{X_{D}=0} \leq$ $e^{-\left\langle u, X_{D}\right\rangle}$, we have $\tilde{u} \leq V_{D}(u) \leq v$, where $v$ is given by (8.4). Therefore Lemma 8.3 follows from Lemma 8.2.

8.3. Proof of Theorem 8.1. Put $\tilde{u}=Q_{\Gamma}(u)$ and $v=Q_{B}(\tilde{u})$. We need to prove that $v=0$. By Theorem 2.1, it is sufficient to show that

$$
v^{E}=0 \quad \text { a.s. }
$$

and that $v$ is bounded. By Lemma 8.3, (8.6) holds for sufficiently small $\delta>0$. By 5.2.B, $v \leq \tilde{u}$ and therefore $v^{E}=0$ a.s. on $\left\{\xi_{\zeta-\notin} \notin V_{\delta}(\Gamma)^{*}\right.$. By the same lemma, if $\delta$ is sufficiently small, then $v^{E}=0$ a.s. on $\left\{\xi_{\zeta-} \notin V_{\delta}(B)^{*}\right\}$. Condition (8.7) is satisfied because $V_{\delta}(\Gamma)^{*}$ and $V_{\delta}(B)^{*}$ are disjoint if $\delta<d(B, \Gamma) / 2$.

Denote by $B_{1}$ the set of all $x \in E^{\prime}$ such that $d(x, B) \leq d(B, \Gamma) / 2$, and let $\Gamma_{1}$ be the closure of $E^{\prime} \backslash B_{1}$. Since $B_{1} \cap \Gamma=\emptyset$, by Lemma 8.3, $\tilde{u}$ is bounded in $V_{\delta}\left(B_{1}\right)$ 
for sufficiently small $\delta$ and the same is true for $v$. Since $B \cap \Gamma_{1}=\emptyset, v$ is bounded in $V_{\delta}\left(\Gamma_{1}\right)$ for sufficiently small $\delta$. Since $B_{1} \cup \Gamma_{1}=E^{\prime}, v$ is bounded in $V_{\delta}\left(E^{\prime}\right)$. The mean value property 2.1.D and Jensen's inequality imply that $v$ is bounded in E.

8.4. Barriers. A barrier in an open set $D \subset E$ is a positive function $u$ such that

$$
L u \leq u^{\alpha} \text { on } D
$$

and, for all $x \in D$,

$$
u^{D}=\infty \quad \Pi_{x} \text {-a.s. on }\{\tau<\zeta\},
$$

where $\tau$ is the first exit time from $D$. Condition (8.9) holds, in particular, if

$$
u=\infty \text { on } \partial D \text {. }
$$

Theorem 8.2. If $u$ is a barrier in $D$, then

$$
v(x)=-\log P_{x}\left\{X_{D}=0\right\} \leq u(x) \quad \text { for all } x \in D .
$$

Proof. Note that $v_{k} \uparrow v$, where

$$
v_{k}(x)=-\log P_{x} e^{-k\left\langle 1, X_{D}\right\rangle} .
$$

Therefore it is sufficient to prove that $v_{k} \leq u$ for all $k$. By Jensen's inequality, $v_{k} \leq k h$, where $h(x)=P_{x}\left\langle 1, X_{D}\right\rangle=\Pi_{x}\{\tau<\zeta\}$ (cf. the proof of Lemma 8.2). For every $x \in D$, by Lemma 2.1 ,

$$
h^{D}=1 \quad \text { a.s. on }\{\tau<\zeta\}, \quad h^{D}=0 \quad \text { a.s. on }\{\tau=\zeta\}
$$

and therefore

$$
v_{k}^{D} \leq 1 \quad \text { a.s. on }\{\tau<\zeta\}, \quad v_{k}^{D}=0 \quad \text { a.s. on }\{\tau=\zeta\} .
$$

On the other hand, by (8.9),

$$
u^{D}=\infty \quad \text { a.s. on }\{\tau<\zeta\}, \quad u^{D} \geq 0 \quad \text { a.s. on }\{\tau=\zeta\} .
$$

By (8.12) and (8.13), $\left(v_{k}-u\right)^{D} \leq 0 \Pi_{x}$-a.s. Since $v_{k}-u \leq k$ and $L u-u^{\alpha} \leq 0=$ $L v_{k}-v_{k}^{\alpha}, v_{k} \leq u$ in $D$ by Theorem 2.1 .

Corollary. A pair $(c, D)$, where $c \in D^{*}$, is admissible if there exists a barrier $u$ in $D$ that is bounded in $V_{\delta}(c)$ for some $\delta>0$.

8.5. Bounded domains in $\mathbb{R}^{d}$. Now we investigate $(L, \alpha)$-superdiffusion under the following assumptions:

8.5.A. $E$ is a bounded domain in $\mathbb{R}^{d}$.

8.5.B. The coefficients of the operator

$$
L=\sum_{i, j=1}^{d} a_{i j}(x) \frac{\partial^{2}}{\partial x_{i} \partial x_{j}}+\sum_{i=1}^{d} b_{i}(x) \frac{\partial}{\partial x_{i}}
$$

satisfy the following conditions: there exist constants $K_{1}, K_{2}$ such that

$$
0<\sum_{i, j=1}^{d} a_{i j}(x) \lambda_{i} \lambda_{j} \leq K_{1} \sum_{i=1}^{d} \lambda_{i}^{2}
$$

and

$$
\sum_{i=1}^{d} b_{i}(x)^{2} \leq K_{2}
$$


for all $x \in D$ and all $\lambda_{1}, \ldots, \lambda_{d} \in \mathbb{R}$.

We use the notation $d_{0}(x, y)$ for the Euclidean distance between $x$ and $y$. We denote by $\partial E$ the boundary of $E$ in the Euclidean topology of $\mathbb{R}^{d}$, and we put $U_{\varepsilon}(B)=\left\{x \in E: d_{0}(x, B)<\varepsilon\right\}$ for an arbitrary $B \subset \partial E$. We say that points $c \in$ $E^{\prime}$ and $b \in \partial E$ are associated and we write $c \hookrightarrow b$ if there exists a sequence $x_{n} \in E$ such that $d\left(x_{n}, c\right) \rightarrow 0$ and $d_{0}\left(x_{n}, b\right) \rightarrow 0$. Note that the set $C_{b}=\left\{c \in E^{\prime}: c \hookrightarrow b\right\}$ is $M$-closed, and the set $B_{c}=\{b \in \partial E: c \hookrightarrow b\}$ is closed in the Euclidean topology.

Theorem 8.3. Under conditions 8.5.A, B, the pair $\left.\left(c, U_{\varepsilon}\left(B_{c}\right)\right)\right)$ is admissible for every $c \in E^{\prime}$.

The proof of Theorem 8.3 is based on the following lemmas.

Lemma 8.4. For every $\varepsilon>0$ and every $b \in \partial E$, there exists a constant $\lambda$ such that

$$
v_{\varepsilon}(x)=\lambda\left[\varepsilon^{2}-d_{0}(x, b)^{2}\right]^{-2 /(\alpha-1)}
$$

is a barrier in $U_{\varepsilon}(b)$.

Proof. Clearly, $v_{\varepsilon}$ satisfies condition (8.10). A direct computation (see [3, p.102]) shows that $L v_{\varepsilon}-v_{\varepsilon}^{\alpha} \leq 0$ for sufficiently large $\lambda$.

Lemma 8.5. For every $\varepsilon>0$, there exists $\delta>0$ such that $V_{\delta}(c) \subset U_{\varepsilon}\left(B_{c}\right)$.

Proof. If this is false, then there exist $\varepsilon>0$ and $x_{n} \in E$ such that $d\left(x_{n}, c\right)<1 / n$ but $d_{0}\left(x_{n}, B_{c}\right)>\varepsilon$. Choose a subsequence $x_{n_{k}}$ which converges in the Euclidean topology. Clearly, its limit $b$ belongs to $B_{c}$, in contradiction to the inequality $d_{0}\left(x_{n_{k}}, B_{c}\right)>\varepsilon$.

Proof of Theorem 8.3. For every $b \in B_{c}, U_{\varepsilon}(b) \subset U_{\varepsilon}\left(B_{c}\right)$. Hence

$$
u(x)=-\log P_{x}\left\{X_{U_{\varepsilon}\left(B_{c}\right)}=0\right\}
$$

is dominated by

$$
v^{b}(x)=-\log P_{x}\left\{X_{U_{\varepsilon}(b)}=0\right\} .
$$

By Theorem 8.2 and Lemma 8.4, $v^{b}$ does not exceed the barrier $v_{\varepsilon}$ defined by (8.14). We conclude that $u$ is bounded on $U_{\varepsilon / 2}(b)$ for every $b \in B_{c}$. Since $B_{c}$ is compact, it contains a finite subset $\left\{b_{1}, \ldots, b_{n}\right\}$ such that, for every $b \in B_{c}$, $\min _{i} d_{0}\left(b, b_{i}\right)<\varepsilon / 4$. Therefore $U_{\varepsilon / 4}\left(B_{c}\right) \subset U_{\varepsilon / 2}\left(b_{1}\right) \cup \cdots \cup U_{\varepsilon / 2}\left(b_{n}\right)$. By Lemma 8.5, there exists $\delta>0$ such that $V_{\delta}(c) \subset U_{\varepsilon / 4}\left(B_{c}\right)$. Hence $u$ is bounded on $V_{\delta}(c)$.

\section{6.}

Theorem 8.4. A point $c \in E^{\prime}$ is normal if $B_{c} \cap B_{c}^{\prime}=\emptyset$ for all $c^{\prime} \neq c$.

Proof. It follows from Theorem 8.3 that $c$ is normal if, for every $\varepsilon>0$, there exists $\delta>0$ such that $U_{\delta}\left(B_{c}\right) \subset V_{\varepsilon}(c)$. Suppose that this condition is not satisfied. Then there exist $\varepsilon>0$ and a sequence $x_{n} \in E$ such that $d_{0}\left(x_{n}, B_{c}\right)<1 / n$ and $d\left(x_{n}, c\right) \geq \varepsilon$. Choose a subsequence $x_{n_{k}}$ which converges relative to both metrics $d_{0}$ and $d$. Denote by $b$ and $c^{\prime}$ its Euclidean limit and $M$-limit. Clearly, $b \in B_{c} \cap B_{c^{\prime}}$ and $d\left(c^{\prime}, c\right) \geq \varepsilon$.

Theorem 8.5. A point $c \in E^{\prime}$ is normal if $B_{c}$ consists of a single point $b$ and if $c$ is an isolated point in $C_{b}$. 
First we establish two lemmas.

Lemma 8.6. For every $\varepsilon>0$, there exists $\delta>0$ such that $U_{\delta}(b) \subset V_{\varepsilon}\left(C_{b}\right)$.

The proof is similar to the proof of Lemma 8.5.

Lemma 8.7. Suppose $D_{1}, D_{2}$ are two disjoint open subsets of $E$ and $D=D_{1} \cup D_{2}$. Then, for every $x \in D_{1}, P_{x}\left\{X_{D_{1}}=X_{D}\right\}=1$.

Proof. By Lemma 3.1 in [9, p.1978], $X_{D}(\Gamma) \geq X_{D_{1}}(\Gamma) P_{x^{-a}}$.s. for every $\Gamma \subset D$. By (1.13), $P_{x} X_{D}(\Gamma)=\Pi_{x}\left\{\xi_{\tau} \in \Gamma\right\}$ and $P_{x} X_{D_{1}}(\Gamma)=\Pi_{x}\left\{\xi_{\tau_{1}} \in \Gamma\right\}$, where $\tau, \tau_{1}$ are the first exit times from $D$ and from $D_{1}$. If $x \in D_{1}$, then $\tau=\tau_{1} \Pi_{x}$-a.s., and therefore $P_{x} X_{D}(\Gamma)=P_{x} X_{D_{1}}(\Gamma)$.

Proof of Theorem 8.5. It is sufficient to prove that the pair $\left(c, V_{\varepsilon}(c)\right)$ is admissible for all sufficiently small $\varepsilon$. There exists $\beta>0$ such that $d\left(c, c^{\prime}\right)>2 \beta$ for all $c^{\prime} \in C(b), c^{\prime} \neq c$. Suppose that $\varepsilon<\beta$. Then $V_{\varepsilon}\left(C_{b}\right)=V_{\varepsilon}(c) \cup \tilde{V}$, where $\tilde{V}$ is open and disjoint from $V_{\varepsilon}(c)$. By Lemma 8.6, $U_{\delta}(b) \subset V_{\varepsilon}\left(C_{b}\right)$ for some $\delta$. By Theorem 8.3, $\left(c, U_{\delta}\left(B_{c}\right)\right)$ is admissible. Hence $\left(c, U_{\delta}(b)\right)$ and $\left(c, V_{\varepsilon}\left(C_{b}\right)\right)$ are also admissible. It remains to note that, by Lemma 8.7, $X_{V_{\varepsilon}\left(C_{b}\right)}=X_{V_{\varepsilon}(c)} P_{x^{-a} \text {.s. for }}$ all $x \in V_{\varepsilon}(c)$.

\section{REFERENCES}

1. M. Brelot, On Martin boundaries, Unpublished lectures, Hiroshima University, 1962; Russian translation in Matematika 9:5 (1965),136-155.

2. E. B. Dynkin, Markov Processes, Springer-Verlag, Berlin, Heidelberg, 1965. MR 33:1887

3. __ A probabilistic approach to one class of nonlinear differential equations, Probab. Th. Rel. Fields 89 (1991), 89-115. MR 92d:35090

4. - Superdiffusions and parabolic nonlinear differential equations, Ann. Probab. 20 (1992), 942-962. MR 93d:60124

5. , Superprocesses and partial differential equations, Ann. Probab. 21 (1993), 11851262. MR 94j:60156

6. - A probabilistic approach to a nonlinear differential equation on a Riemannian manifold, Teoriya Veroyatnostei i ee Primeneniya 42 (1997), 336-341; English transl., to appear in Theory Probab. Appl. 42 (1997). CMP 98:02

7. E. B. Dynkin and S. E. Kuznetsov, Superdiffusions and removable singularities for quasilinear partial differential equations, Comm. Pure \& Appl. Math 49 (1996), 125-176. MR 97m:60114

8. , Solutions of $L u=u^{\alpha}$ dominated by L-harmonic functions, Journale d'Analyse Math. 68 (1996), 15-37. MR 97f:35048

9. - Linear additive functionals of superdiffusions and related nonlinear P.D.E., Trans. Amer. Math. Soc. 348 (1996), 1959-1987. MR 97d:60135

10. , Nonlinear parabolic P.D.E. and additive functionals of superdiffusions, Ann. Probab. 25 (1997), 662-701. CMP 97:08

11. _ Natural linear additive functionals of superprocesses, Ann. Probab. 25 (1997), 640661. CMP 97:08

12. Math. Soc. 350 (1998), 4499-4519.

13. J.F. Le Gall, Solutions positives de $\Delta u=u^{2}$ dans le disque unité, C.R. Acad. Sci. Paris, Série I 317 (1993), 873-878. MR 94h:35059

14. _ A probabilistic Poisson representation for positive solutions of $\Delta u=u^{2}$ in a planar domain, Comm. Pure Appl. Math. 50 (1997), 69-103. MR 88e:60144

15. M. Marcus and L. Véron, Trace au bord des solutions positives d'équations elliptiques non linéaires, C.R. Acad.Sci Paris ser I 321 (1995), 179-184. MR 96f:35045

16. _ Trace au bord des solutions positives d'équations elliptiques non linéaires. Résultats d'existence and d'unicité, C.R. Acad.Sci Paris ser I 323 (1996), 603-608. MR 97f:35012 
17. _ The boundary trace of positive solutions of semilinear elliptic equations I: The subcritical case. Arch. Rational Mech. Anal., 1998, to appear.

17a. M. Marcus and L. Véron, The boundary trace of positive solutions of semilinear elliptic equations II: The supercritical case. Preprint, 1997.

18. V. G. Maz'ya, Beurling's theorem on a minimum principle for positive harmonic functions, [First published (in Russian) in Zapiski Nauchnykh Seminarov Leningradskogo Otdeleniya Mat. Inst. im. V. A. Steklova 30 (1972), 76-90], J.Soviet Math. 4, 367-379. MR 48:8821

19. K.Yosida, The fundamental solution of the parabolic equation in a Riemannian space, Osaka Math. J. 5:1 (1953), 65-74. MR 15:36b

Department of Mathematics, White Hall, Cornell University, Ithaca, New York 14853-7901

E-mail address: ebd1@cornell.edu

Central Econ.-Math. Institute, Russian Academy of Sciences, 32 Krasikowa, Moscow 117418, Russia

Current address: Department of Mathematics, University of Colorado at Boulder, Boulder, Colorado 80309-0395

E-mail address: sk47@cornell.edu 